\title{
ON SUPERSPECIAL ABELIAN SURFACES AND TYPE NUMBERS OF TOTALLY DEFINITE QUATERNION ALGEBRAS
}

\author{
JIANGWEI XUE AND CHIA-FU YU
}

\begin{abstract}
In this paper we determine the number of endomorphism rings of superspecial abelian surfaces over a field $\mathbb{F}_{q}$ of odd degree over $\mathbb{F}_{p}$ in the isogeny class corresponding to the Weil $q$-number $\pm \sqrt{q}$. This extends earlier works of T.-C. Yang and the present authors on the isomorphism classes of these abelian surfaces, and also generalizes the classical formula of Deuring for the number of endomorphism rings of supersingular elliptic curves. Our method is to explore the relationship between the type and class numbers of the quaternion orders concerned. We study the Picard group action of the center of an arbitrary $\mathbb{Z}$-order in a totally definite quaternion algebra on the ideal class set of said order, and derive an orbit number formula for this action. This allows us to prove an integrality assertion of Vignéras [Enseign. Math. $(2), 1975]$ as follows. Let $F$ be a totally real field of even degree over $\mathbb{Q}$, and $D$ be the (unique up to isomorphism) totally definite quaternion $F$-algebra unramified at all finite places of $F$. Then the quotient $h(D) / h(F)$ of the class numbers is an integer.
\end{abstract}

\section{INTRODUCTION}

Throughout this paper $p$ denotes a prime number. Let $D_{p, \infty}$ denote the unique definite quaternion $\mathbb{Q}$-algebra up to isomorphism ramified exactly at $p$ and $\infty$. The classical result of Deuring establishes a bijection between the set $\Lambda_{p}$ of isomorphism classes of supersingular elliptic curves over $\overline{\mathbb{F}}_{p}$ and the set $\operatorname{Cl}\left(D_{p, \infty}\right)$ of ideal classes of a maximal order in $D_{p, \infty}$. The class number $h$ of $D_{p, \infty}$ is well known due to Eichler [6] (Deuring and Igusa gave different proofs of this result), and is given by

$$
h=\frac{p-1}{12}+\frac{1}{3}\left(1-\left(\frac{-3}{p}\right)\right)+\frac{1}{4}\left(1-\left(\frac{-4}{p}\right)\right),
$$

where $(\dot{\bar{p}})$ denotes the Legendre symbol. Under the correspondence $\Lambda_{p} \simeq \operatorname{Cl}\left(D_{p, \infty}\right)$, the type number $t$ of $D_{p, \infty}$ is equal to the number of non-isomorphic endomorphism rings of members $E$ in $\Lambda_{p}$. An explicit type formula is also well known due to Deuring [5], which is given by

$$
t=\frac{p-1}{24}+\frac{1}{6}\left(1-\left(\frac{-3}{p}\right)\right)+\left\{\begin{array}{lll}
h(-p) / 4 & \text { if } p \equiv 1 & (\bmod 4) \\
1 / 4+h(-p) / 2 & \text { if } p \equiv 7 & (\bmod 8) \\
1 / 4+h(-p) & \text { if } p \equiv 3 & (\bmod 8)
\end{array}\right.
$$

Date: September 13, 2018.

2010 Mathematics Subject Classification. 11R52, 11G10.

Key words and phrases. superspecial abelian surfaces, type numbers, class numbers, totally definite quaternion algebras. 
for $p>3$, and $t=1$ for $p=2,3$. Here for any square-free integer $d \in \mathbb{Z}$, we write $h(d)$ for the class number of $\mathbb{Q}(\sqrt{d})$. Though these classical results were well established by 1950, different proofs with various ingredients such as mass formulas, Tamagawa numbers, theta series, cusp forms, algebraic modular forms, Atkin-Lehner involutions and traces of Hecke operators, have been generalized and revisited many times even until now. Different angles and approaches such as the Eichler-Shimizu-Jacquet-Langlands correspondence and trace formulas also play important roles in the development. This paper is one instance of them, where we would like to generalize the explicit formulas (1.1) and (1.2) from $D_{p, \infty}$ to $D_{p, \infty} \otimes \mathbb{Q}(\sqrt{p})$, which is the unique totally definite quaternion $\mathbb{Q}(\sqrt{p})$-algebra (up to isomorphism) unramified at all finite places. Recall that a quaternion algebra $D$ over a totally real number field $F$ is said to be totally definite if $D \otimes_{F, \sigma} \mathbb{R}$ is isomorphic to the Hamilton quaternion algebra $\mathbb{H}$ for every embedding $\sigma: F \hookrightarrow \mathbb{R}$. Our interest for totally definite quaternion algebras stems from the study abelian varieties over finite fields. Note that $D_{p, \infty}$ and $D_{p, \infty} \otimes \mathbb{Q}(\sqrt{p})$ are the only two algebras that can occur as the endomorphism algebra of an abelian variety over a finite field [31, but do not satisfy the Eichler condition [27, Definition 34.3]. The cases where the endomorphism algebras satisfy the Eicher condition are easier to treat. Indeed, by a result of Jacobinski [16, Theorem 2.2], the class number of an order in such an algebra is equal to a ray class number of its center. Observe that the number $h$ in (1.1) (resp. $t$ in (1.2) ) is also equal to the number of $\mathbb{F}_{q}$-isomorphism classes (resp. non-isomorphic endomorphism rings) of supersingular elliptic curves in the isogeny class corresponding to the Weil $q$-number $\sqrt{q}$ (or $-\sqrt{q}$ ), for any field $\mathbb{F}_{q}$ containing $\mathbb{F}_{p^{2}}$. Thus, the present work may be also reviewed as an generalization of explicit formulas (1.1) and (1.2) in the arithmetic direction. A generalization of the geometric direction in the sense that the set $\Lambda_{p}$ is replaced by the superspecial locus of the Siegel moduli spaces has been inverstigated by Hashimoto, Ibukiyama, Katsura and Oort [10,11, 12,14,17.

Now let $q$ be an odd power of $p$, and let $\operatorname{Sp}(\sqrt{q})$ be the set of isomorphism classes of superspecial abelian surfaces over $\mathbb{F}_{q}$ in the isogeny class corresponding to the Weil number $\pm \sqrt{q}$. As a generalization of (1.1), T.-C. Yang and the present authors [37, Theorem 1.2] (also see [39, Theorem 1.3]) show the following explicit formula for $|\operatorname{Sp}(\sqrt{q})|$.

Theorem 1.1. Let $F=\mathbb{Q}(\sqrt{p})$, and $O_{F}$ be its ring of integers.

(1) The cardinality of $\operatorname{Sp}(\sqrt{q})$ depends only on $p$, and is denoted by $H(p)$.

(2) We have $H(p)=1,2,3$ for $p=2,3,5$, respectively.

(3) For $p>5$ and $p \equiv 3(\bmod 4)$, one has

$$
H(p)=h(F)\left[\frac{\zeta_{F}(-1)}{2}+\left(13-5\left(\frac{2}{p}\right)\right) \frac{h(-p)}{8}+\frac{h(-2 p)}{4}+\frac{h(-3 p)}{6}\right],
$$

where $h(F)$ is the class number of $F$ and $\zeta_{F}(s)$ is the Dedekind zeta function of $F$.

(4) For $p>5$ and $p \equiv 1(\bmod 4)$, one has

$H(p)=\left\{\begin{array}{lll}h(F)\left[8 \zeta_{F}(-1)+h(-p) / 2+\frac{2}{3} h(-3 p)\right] & \text { for } p \equiv 1 & (\bmod 8) ; \\ h(F)\left[\left(\frac{45+\varpi}{2 \varpi}\right) \zeta_{F}(-1)+\left(\frac{9+\varpi}{8 \varpi}\right) h(-p)+\frac{2}{3} h(-3 p)\right] & \text { for } p \equiv 5 & (\bmod 8) ;\end{array}\right.$

where $\varpi:=\left[O_{F}^{\times}: A^{\times}\right] \in\{1,3\}$ and $A:=\mathbb{Z}[\sqrt{p}]$ is the suborder of index 2 in $O_{F}$.

Let $\mathcal{T}(\operatorname{Sp}(\sqrt{q}))$ denote the set of isomorphism classes of endomorphism rings of abelian surfaces in $\operatorname{Sp}(\sqrt{q})$. The cardinality of $\mathcal{T}(\operatorname{Sp}(\sqrt{q}))$ again depends only on 
the prime $p$ ( [39, Theorem 1.3], see also Sect. 3), and is denoted by $T(p)$. In this paper we give an explicit formula for $T(p)$, which generalizes (1.2).

Theorem 1.2. Let $F=\mathbb{Q}(\sqrt{p})$ and $T(p):=|\mathcal{T}(\operatorname{Sp}(\sqrt{q}))|$.

(1) We have $T(p)=1,2,3$ for $p=2,3,5$, respectively.

(2) For $p \equiv 3(\bmod 4)$ and $p \geq 7$, we have

$$
T(p)=\frac{\zeta_{F}(-1)}{2}+\left(13-5\left(\frac{2}{p}\right)\right) \frac{h(-p)}{8}+\frac{h(-2 p)}{4}+\frac{h(-3 p)}{6} .
$$

(3) For $p \equiv 1(\bmod 4)$ and $p \geq 7$, we have

$$
T(p)=8 \zeta_{F}(-1)+\frac{1}{2} h(-p)+\frac{2}{3} h(-3 p) .
$$

It follows from Theorems 1.1 and 1.2 that $H(p)=T(p) h(F)$ except for the case where $p \equiv 5(\bmod 8)$ and $\varpi=1$. When $p \equiv 3(\bmod 4)$, we actually prove this result first and use it to get formula (1.3). For the case where $p \equiv 1(\bmod 4)$, we explain how this coindence arises in part (1) of Remark 4.3.

Similar to Deuring's result for supersingular elliptic curves, the proof of Theorem 1.2 is reduced to the calculation of the type number of a maximal order $\mathbb{O}_{1}$ in in $D_{p, \infty} \otimes \mathbb{Q}(\sqrt{p})$, as well as those of certain proper $\mathbb{Z}[\sqrt{p}]$-orders $\mathbb{O}_{8}$ and $\mathbb{O}_{16}$ (see (4.1) and (4.2) for definition of these orders). We recall briefly the concept of proper $A$-orders. Henceforth all orders are assumed to be of full rank in their respective ambient algebras. Let $F$ be a number field, and $A$ be a $\mathbb{Z}$-order in $F$ (not necessarily maximal). A $\mathbb{Z}$-order $\mathcal{O}$ in a finite dimensional semisimple $F$-algebra $\mathscr{D}$ is said to be a proper $A$-order if $\mathcal{O} \cap F=A$. Unlike [4, Definition 23.1], we do not require $\mathcal{O}$ to be a projective $A$-module. The class number of $\mathcal{O}$ is denoted by $h(\mathcal{O})$. When $F$ is a totally real number field, a proper $A$-order in a CM-extension (i.e. a totally imaginary quadratic extension) of $F$ is call a $C M$ proper $A$-order. If $A$ coincides with $O_{F}$, then we drop the adjective "proper" and simply write " $O_{F}$-order".

It is well known that the type number of a totally definite Eichler order of square-free level can be calculated by Eichler's trace formula [7] see also [18] for general $O_{F}$-orders. Some errors of Eichler's formula were found and corrected later independently by M. Peters 23. and by A. Pizer 24. Eichler's trace formula contains a number of data which are generally not easy to compute. In [33. p. 212] M.-F, Vignéras gave an explicit formula for the type number of any totally definite quaternion algebra $D$, over any real quadratic field $F=\mathbb{Q}(\sqrt{d})$, that is unramified at all finite places. Her formula was based on the explicit formula for the class number $h(D)$ in [32, Theorem 3.1] and the class-type number relationship $h(D)=$ $t(D) h(F)$; see [33, p. 212]. Unfortunately, it was pointed out by Ponomarev in [26, Concluding remarks, p. 103] that the formula in [33. p. 212] is not a formula for $t(D)$ in general. This conclusion is based on his explicit calculations for class numbers of positive definite quaternary quadratic forms [26, Sect. 5], and the correspondence between quaternary quadratic forms and types of quaternion algebras established in [25, Sect. 4]. The source of the difficulty is that the class-type number relationship $h(D)=t(D) h(F)$ may fail in general], even if $D$ is unramified at all finite places. To remedy this, we examine more closely the Picard group action described below.

\footnotetext{
${ }^{1}$ In [26, p. 103] it reads "the class number of $\mathfrak{A}_{K}$ divided by the proper class number of $K$ is not, in general, the type number $t$ ". However it should read "the class number" instead of "the proper class number" as the former one is what Vignéras' formula is based on.
} 
Let $F$ be an arbitrary number field, $A \subseteq O_{F}$ a $\mathbb{Z}$-order in $F$, and $\mathcal{O}$ a proper $A$-order in a quaternion $F$-algebra $D$. The Picard group $\operatorname{Pic}(A)$ acts naturally on the finite set $\mathrm{Cl}(\mathcal{O})$ of locally principal right ideal classes of $\mathcal{O}$ by the map

$$
\operatorname{Pic}(A) \times \mathrm{Cl}(\mathcal{O}) \rightarrow \mathrm{Cl}(\mathcal{O}), \quad([\mathfrak{a}],[I]) \mapsto[\mathfrak{a} I],
$$

where $\mathfrak{a}$ (resp. $I$ ) denotes a locally principal fractional $A$-ideal in $F$ (resp. right $\mathcal{O}$-ideal in $D$ ), and $[\mathfrak{a}]$ (resp. $[I]$ ) denotes its ideal class. Let $\overline{\mathrm{Cl}}(\mathcal{O})$ be the set of orbits of this action, and $r(\mathcal{O})$ be its cardinality:

$$
r(\mathcal{O}):=|\overline{\mathrm{Cl}}(\mathcal{O})|=|\operatorname{Pic}(A) \backslash \mathrm{Cl}(\mathcal{O})| .
$$

One of main results of the this paper is a formula for $r(\mathcal{O})$ when $D$ is a totally definite quaternion algebra over a totally real field.

Theorem 1.3. Let $A$ and $\mathcal{O}$ be as above. Suppose that $F$ is a totally real number field, and $D$ is a totally definite quaternion $F$-algebra. The number of orbits of the Picard group action (1.5) can be calculated by the following formula:

$$
r(\mathcal{O})=\frac{1}{h(A)}\left(h(\mathcal{O})+\sum_{1 \neq[\mathfrak{a}] \in \mathfrak{C}_{2}(A, \widetilde{A})} \sum_{\llbracket B \rrbracket \in \mathscr{B}_{[\mathfrak{a}]}} \frac{1}{2}(2-\delta(B)) h(B) \prod_{\ell} m_{\ell}(B)\right),
$$

where we refer to (3.8), (3.5), (3.3), (3.14) for the definitions of $\mathfrak{C}_{2}(A, \widetilde{A}), \mathscr{B}_{[\mathfrak{a}]}$, $\delta_{B}, m_{\ell}(B)$ respectively.

If $A=O_{F}$, then the formula for $r(\mathcal{O})$ can be simplified further (see Theorem 3.7).

Assume that $F$ is a totally real field of even degree over $\mathbb{Q}$, and $D$ is the unique up to isomorphism quaternion $F$-algebra unramified at all finite places of $F$. Let $\mathcal{O}$ be a maximal $O_{F}$-order in $D$. Then $t(D)=r(\mathcal{O})$, and hence Theorem 1.3 leads to a type number formula for such $D$ in Corollary 3.11] In [32, Remarque, p. 82], Vigénras asserted that $h(D) / h(F)$ is always an integer. However, the assertion was mixed with the misconception that $h(D)=t(D) h(F)$ holds unconditionally on $F$, and we could not locate a precise proof of this integrality elsewhere. As an application of our orbit number formula, we prove in Theorem 5.4 that $h(D) / h(F) \in \mathbb{N}$ for all $F$. On the other hand, we give in Corollary 3.5 a necessary and sufficient condition on $F$ such that the relationship $h(D)=h(F) t(D)$ remains valid. In particular, for real quadratic fields satisfying this condition, Vigénras's formula [33, p. 212] does give a formula for the type number $t(D)$. This approach of calculating the type number via the class number also paves the way to our proof of Theorem 1.2 , where we treat certain orders (namely, $\mathbb{O}_{8}$ and $\mathbb{O}_{16}$ ) that does not contain $O_{F}$. The type numbers of such orders are not covered by previous methods of Eichler-Pizer and Ponomarev.

This paper is organized as follows. In Section 2 we provide some preliminary studies on the $\operatorname{Pic}(A)$-action on $\operatorname{Cl}(\mathcal{O})$. This is carried out more in depth for totally definite quaternion algebras in Section 3. and we derive the orbit number formula and its corollaries there. The calculations for Theorem 1.2 are worked out in Section 4, and we prove the integrality of $h(D) / h(F)$ in Section 5 .

\section{Preliminaries on the Picard group action}

Let $F$ be a number field, $O_{F}$ its ring of integers, and $A \subseteq O_{F}$ a $\mathbb{Z}$-order in $F$. Let $D$ be a quaternion $F$-algebra and $\mathcal{O}$ a proper $A$-order in $D$. This section provides a preliminary study of the $\operatorname{Pic}(A)$-action on $\operatorname{Cl}(\mathcal{O})$ in (1.5). 
We follow the notation of [37, Sections 2.1-2.3]. Recall that $D$ admits a canonical involution $x \mapsto \bar{x}$ such that $\operatorname{Tr}(x)=x+\bar{x}$ and $\operatorname{Nr}(x)=x \bar{x}$ are respectively the reduced trace and reduced norm of $x \in D$. The reduced discriminant $\mathfrak{d}(D)$ of $D$ is the product of all finite primes of $F$ that are ramified in $D$. Let $\widetilde{A}:=\operatorname{Nr}_{A}(\mathcal{O})$ be the norm of $\mathcal{O}$ over $A$. More explicitly, $\widetilde{A}$ is the $A$-submodule of $O_{F}$ spanned by the reduced norms of elements of $\mathcal{O}$. Clearly, $\widetilde{A}$ is closed under multiplication, hence a suborder of $O_{F}$ containing $A$. By [37, Lemma 3.1.1], $\mathcal{O}$ is closed under the canonical involution if and only if $\widetilde{A}=A$. In particular, any $O_{F}$-order in $D$ is closed under the canonical involution. We have a the natural surjective map between the Picard groups:

$$
\pi: \operatorname{Pic}(A) \rightarrow \operatorname{Pic}(\widetilde{A}), \quad \mathfrak{a} \mapsto \mathfrak{a} \widetilde{A} .
$$

Given an ideal class $[I] \in \mathrm{Cl}(\mathcal{O})$, we study the stabilizer $\operatorname{Stab}([I]) \subseteq \operatorname{Pic}(A)$ of the $\operatorname{Pic}(A)$ action on $\mathrm{Cl}(\mathcal{O})$ as in (1.5). Let $I^{-1}$ be the inverse of $I$, and $\mathcal{O}_{l}(I)=\{x \in$ $D \mid x I \subseteq I\}$ the associated left order of $I$. We have $\mathcal{O}_{l}(I)=I I^{-1}$ [34, Section I.4]. Thus

$$
[\mathfrak{a} I]=[I] \quad \text { if and only if } \quad\left[\mathfrak{a} \mathcal{O}_{l}(I)\right]=\left[\mathcal{O}_{l}(I)\right],
$$

where $\left[\mathcal{O}_{l}(I)\right]$ is the trivial ideal class of $\mathcal{O}_{l}(I)$. Therefore, the study of $\operatorname{Stab}([I])$ often reduces to that of $\operatorname{Stab}([\mathcal{O}])$. We have

$$
\operatorname{Stab}([\mathcal{O}])=\{[\mathfrak{a}] \in \operatorname{Pic}(A) \mid \exists \lambda \in D \text { such that } \mathfrak{a} \mathcal{O}=\lambda \mathcal{O}\} .
$$

Since $\mathfrak{a} \mathcal{O}$ is a nonzero two-sided $\mathcal{O}$-ideal, $\lambda$ lies in the normalizer $\mathcal{N}(\mathcal{O}) \subseteq D^{\times}$ by [34, Exercise I.4.6]. To study more closely the relationship between $\mathfrak{a}$ and $\lambda$, we make use of the following lemma from commutative algebra.

Lemma 2.1. Let $R \subseteq S$ be an extension of unital rings, with $R$ commutative and $S$ a finite $R$-module.

(i) If $\mathfrak{c} \subseteq R$ is an $R$-ideal with $\mathfrak{c} S=S$, then $\mathfrak{c}=R$.

(ii) Let $L$ be the totally quotient ring of $R$. Suppose that the natural map $S \rightarrow$ $S \otimes_{R} L$ is injective, and $S \cap L=R$ in $S \otimes_{R} L$. If $\mathfrak{c} \subset L$ is an $R$-submodule with $\mathfrak{c} S=\lambda S$ for some $\lambda \in L^{\times}$, then $\mathfrak{c}=\lambda R$.

Proof. (i) Since $S$ is a finite $R$-module, the equality $\mathfrak{c} S=S$ implies that there exists $a \in \mathfrak{c}$ such that $(1-a) S=0$ by [8, Corollary 4.7]. Necessarily $a=1$ since $1 \in S$, and hence $1 \in \mathfrak{c}$ and $\mathfrak{c}=R$.

(ii) Let $\mathfrak{c}^{\prime}=\frac{1}{\lambda} \mathfrak{c}$. Then $\mathfrak{c}^{\prime} S=S$, which implies that $\mathfrak{c}^{\prime} \subset S$. We have $\mathfrak{c}^{\prime} \subseteq S \cap L=R$, and hence $\mathfrak{c}^{\prime}$ is an integral ideal of $R$. Now it follows from part (i) that $\mathfrak{c}^{\prime}=R$, equivalently, $\mathfrak{c}=\lambda R$.

We return to the study of the $\operatorname{Pic}(A)$-action on $\operatorname{Cl}(\mathcal{O})$.

Corollary 2.2. Suppose that $\mathfrak{a} \subset F$ is a locally principal nonzero fractional A-ideal with $\mathfrak{a} \mathcal{O}=\lambda \mathcal{O}$ for some $\lambda \in D$.

(i) If $\lambda \in F$, then $\mathfrak{a}=\lambda A$.

(ii) If $\lambda \notin F$, then $\mathfrak{a} B=\lambda B$ with $B=F[\lambda] \cap \mathcal{O}$. In particular, [a] belongs to the kernel of the canonical map $\operatorname{Pic}(A) \rightarrow \operatorname{Pic}(B)$.

Proof. Part (i) follows directly from Lemma 2.1(ii) with $R=A, S=\mathcal{O}$ and $\mathfrak{c}=\mathfrak{a}$, and part (ii) follows with $R=B, S=\mathcal{O}$, and $\mathfrak{c}=\mathfrak{a} B$. 
We say a locally principal fractional ideal $\mathfrak{a}$ of $A$ capitulates in $B$ if the extended ideal $\mathfrak{a} B$ is principal. The capitulation problems (for abelian extensions of number fields $K / F$ with $A=O_{F}$ and $B=O_{K}$ ) was studied by Hilbert (see Hilbert Theorem 94), and it continues to be a field of active research up to this day 2, 15,30. We will follow up on this line of investigation in Section 3. particularly in the derivation of the orbit number formula (see Theorem 3.7). However, our result does not explicitly depend on the works just cited.

Lemma 2.3. For any ideal class $[I] \in \mathrm{Cl}(\mathcal{O})$, the stabilizer $\operatorname{Stab}([I])$ is contained in the kernel of the homomorphism

$$
\mathrm{S} q: \operatorname{Pic}(A) \rightarrow \operatorname{Pic}(\widetilde{A}), \quad[\mathfrak{a}] \mapsto[\mathfrak{a} \widetilde{A}]^{2} .
$$

Proof. Clearly, $\mathrm{Nr}_{A}$ commutes with any localization of $A$. Thus $\operatorname{Nr}_{A}\left(\mathfrak{a} \mathcal{O}^{\prime}\right)=$ $\mathfrak{a}^{2} \operatorname{Nr}_{A}\left(\mathcal{O}^{\prime}\right)$ for every proper $A$-order $\mathcal{O}^{\prime}$ and locally principal fractional $A$-ideal a. Since $I$ is locally principal, $\mathcal{O}_{l}(I)$ is a proper $A$-order with $\operatorname{Nr}_{A}\left(\mathcal{O}_{l}(I)\right)=\widetilde{A}$ by [37, Section 2.3]. Suppose that $\mathfrak{a} \mathcal{O}_{l}(I)=\lambda \mathcal{O}_{l}(I)$ for some $\lambda \in D^{\times}$. Taking $\mathrm{Nr}_{A}$ on both sides, we get

$$
\mathfrak{a}^{2} \widetilde{A}=\operatorname{Nr}(\lambda) \widetilde{A}
$$

and hence $[\mathfrak{a}] \in \operatorname{ker}(\mathrm{S} q)$.

Corollary 2.4. Let $\operatorname{Pic}(\widetilde{A})^{2}$ be the subgroup of $\operatorname{Pic}(\widetilde{A})$ consisting of the $\widetilde{A}$-ideal classes that are perfect squares. Then the number $h(\mathcal{O}) /\left|\operatorname{Pic}(\widetilde{A})^{2}\right|$ is an integer.

Proof. The map $\operatorname{Sq}: \operatorname{Pic}(A) \rightarrow \operatorname{Pic}(\widetilde{A})$ factors as $\operatorname{Pic}(A) \rightarrow \operatorname{Pic}(\widetilde{A})^{2} \hookrightarrow \operatorname{Pic}(\widetilde{A})$. By Lemma 2.3, each $\operatorname{Pic}(A)$-orbit of $\operatorname{Cl}(\mathcal{O})$ has cardinality divisible by $\left|\operatorname{Pic}(\widetilde{A})^{2}\right|$. The corollary follows.

Corollary 2.5. Suppose that $\mathcal{O}$ is closed under the canonical involution and $h(A)$ is odd. Then the action of $\operatorname{Pic}(A)$ on $\operatorname{Cl}(\mathcal{O})$ is free.

Proof. As remarked before, $\mathcal{O}$ is closed under the canonical involution if and only if $\widetilde{A}=A$. The two conditions imply that $\operatorname{ker}(\mathrm{S} q)$ is trivial, so the corollary follows from Lemma 2.3

Remark 2.6. See 3, Corollary (18.4), p. 134] for the complete list of all quadratic fields $F=\mathbb{Q}(\sqrt{d})$ with odd class number. When $A \neq \widetilde{A}$, the action of $\operatorname{Pic}(A)$ on $\mathrm{Cl}(\mathcal{O})$ needs not to be free, even if $h(A)$ is odd. For example, let $A=\mathbb{Z}[\sqrt{37}]$ and $\mathcal{O}=\mathbb{O}_{8}$ (see (4.1) and (4.2) for its definition). We have $\widetilde{A}=O_{F}, h(A)=3$ and $h(\mathcal{O})=7$ by [37, (6.10)]. Clearly, the $\operatorname{Pic}(A)$-action on $\mathrm{Cl}(\mathcal{O})$ cannot be free.

In some cases, the Picard group action (1.5) can be utilized to calculated type numbers of certain orders. Concrete examples will be worked out in Section 4 . To explain the ideas, we adopt the adelic point of view. Let $\widehat{\mathbb{Z}}:=\lim \mathbb{Z} / n \mathbb{Z}=\prod_{\ell} \mathbb{Z}_{\ell}$ be the profinite completion of $\mathbb{Z}$, where the product runs over all primes $\ell \in \mathbb{N}$. Given any finite dimensional $\mathbb{Q}$-vector space or finitely generated $\mathbb{Z}$-module $M$, we set $\widehat{M}:=M \otimes_{\mathbb{Z}} \widehat{\mathbb{Z}}$, and $M_{\ell}:=M \otimes_{\mathbb{Z}} \mathbb{Z}_{\ell}$. Two orders $\mathcal{O}_{1}$ and $\mathcal{O}_{2}$ in the quaternion $F$-algebra $D$ are said to be in the same genus if $\widehat{\mathcal{O}}_{1} \simeq \widehat{\mathcal{O}}_{2}$, or equivalently, if there exists $x \in \widehat{D}^{\times}$such that $\widehat{\mathcal{O}}_{1}=x \widehat{\mathcal{O}}_{2} x^{-1}$. They are said to be of the same type if $\mathcal{O}_{2}=x \mathcal{O}_{1} x^{-1}$ for some $x \in D^{\times}$. Let $\mathcal{T}(\mathcal{O})$ denote the set of $D^{\times}$-conjugacy classes of orders in the genus of $\mathcal{O}$, and let $t(\mathcal{O}):=|\mathcal{T}(\mathcal{O})|$ denote the type number of 
$\mathcal{O}$. For example, it is well known that all maximal $O_{F}$-orders of $D$ lie in the same genus. If $\mathcal{O}$ is a maximal $O_{F}$-order, then the set $\mathcal{T}(\mathcal{O})$ (resp. its cardinality $t(\mathcal{O})$ ) depends only on $D$ and is denoted by $\mathcal{T}(D)$ (resp. $t(D)$ ) instead.

Let $\mathcal{N}(\widehat{\mathcal{O}}) \subseteq \widehat{D}^{\times}$be the normalizer of $\widehat{\mathcal{O}}$, which admits a filtration $\mathcal{N}(\widehat{\mathcal{O}}) \unrhd$ $\widehat{F}^{\times} \widehat{\mathcal{O}}^{\times} \unrhd \widehat{\mathcal{O}}^{\times}$. It is easy to see that $\operatorname{Pic}(A) \simeq \widehat{F}^{\times} / F^{\times} \widehat{A}^{\times}$, and

$$
\begin{aligned}
& \mathrm{Cl}(\mathcal{O}) \simeq D^{\times} \backslash \widehat{D}^{\times} / \widehat{\mathcal{O}}^{\times}, \\
& \overline{\mathrm{Cl}}(\mathcal{O}) \simeq D^{\times} \backslash \widehat{D}^{\times} / \widehat{F}^{\times} \widehat{\mathcal{O}}^{\times}, \\
& \mathcal{T}(\mathcal{O}) \simeq D^{\times} \backslash \widehat{D}^{\times} / \mathcal{N}(\widehat{\mathcal{O}}) .
\end{aligned}
$$

Denote the normal subgroup $\mathcal{N}(\mathcal{O}) \cap\left(\widehat{F}^{\times} \widehat{\mathcal{O}}^{\times}\right)$of $\mathcal{N}(\mathcal{O})$ by $\mathcal{N}_{0}(\mathcal{O})$.

Lemma 2.7. Keep the notation of (2.3). There is a canonical isomorphism

$$
\operatorname{Stab}([I]) \simeq \mathcal{N}_{0}\left(\mathcal{O}_{l}(I)\right) / F^{\times} \mathcal{O}_{l}(I)^{\times}, \quad[\mathfrak{a}] \leftrightarrow\left(\lambda \bmod F^{\times} \mathcal{O}_{l}(I)^{\times}\right) .
$$

Proof. In light of (2.2), it is enough to show that $\operatorname{Stab}([\mathcal{O}]) \simeq \mathcal{N}_{0}(\mathcal{O}) / F^{\times} \mathcal{O}^{\times}$, where $[\mathcal{O}] \in \mathrm{Cl}(\mathcal{O})$ is the principal right $\mathcal{O}$-ideal class. Applying the Zassenhaus lemma [28, Lemma 5.49] to $F^{\times} \unlhd D^{\times}$and $\widehat{\mathcal{O}}^{\times} \unlhd \widehat{F}^{\times} \widehat{\mathcal{O}}^{\times}$, we get

$$
\begin{aligned}
\frac{\mathcal{N}_{0}(\mathcal{O})}{F^{\times} \mathcal{O}^{\times}} & =\frac{\left(D^{\times} \cap \widehat{F}^{\times} \widehat{\mathcal{O}}^{\times}\right) F^{\times}}{\left(D^{\times} \cap \widehat{\mathcal{O}}^{\times}\right) F^{\times}} \simeq \frac{\left(D^{\times} \cap \widehat{F}^{\times} \widehat{\mathcal{O}}^{\times}\right) \widehat{\mathcal{O}}^{\times}}{\left(F^{\times} \cap \widehat{F}^{\times} \widehat{\mathcal{O}}^{\times}\right) \widehat{\mathcal{O}}^{\times}}=\frac{\left(D^{\times} \widehat{\mathcal{O}}^{\times} \cap \widehat{F}^{\times}\right) \widehat{\mathcal{O}}^{\times}}{F^{\times} \widehat{\mathcal{O}}^{\times}} \\
& \simeq \frac{D^{\times} \widehat{\mathcal{O}}^{\times} \cap \widehat{F}^{\times}}{\left(D^{\times} \widehat{\mathcal{O}}^{\times} \cap \widehat{F}^{\times}\right) \cap F^{\times} \widehat{\mathcal{O}}^{\times}}=\frac{D^{\times} \widehat{\mathcal{O}}^{\times} \cap \widehat{F}^{\times}}{F^{\times}\left(\widehat{F}^{\times} \cap \widehat{\mathcal{O}}^{\times}\right)}=\frac{D^{\times} \widehat{\mathcal{O}}^{\times} \cap \widehat{F}^{\times}}{F^{\times} \widehat{A}^{\times}}=\operatorname{Stab}([\mathcal{O}]) .
\end{aligned}
$$

We leave it as a routine exercise to check that the adelic isomorphism constructed above matches with the concrete one given in the lemma.

There is a natural surjective map

$$
\Upsilon: \mathrm{Cl}(\mathcal{O}) \rightarrow \mathcal{T}(\mathcal{O}), \quad[I] \mapsto \llbracket \mathcal{O}_{l}(I) \rrbracket,
$$

where $\llbracket \mathcal{O}_{l}(I) \rrbracket$ denotes the $A$-isomorphism class of $\mathcal{O}_{l}(I)$ (equivalently, the $D^{\times}$conjugacy class of $\left.\mathcal{O}_{l}(I)\right)$. Clearly, $\Upsilon$ factors through the projection $\mathrm{Cl}(\mathcal{O}) \rightarrow \overline{\mathrm{Cl}}(\mathcal{O})$.

Proposition 2.8. If $\mathcal{N}(\widehat{\mathcal{O}})=\widehat{F}^{\times} \widehat{\mathcal{O}}^{\times}$, then $t(\mathcal{O})=r(\mathcal{O})$. If further $\mathcal{O}$ is stable under the canonical involution and $h(A)$ is odd, then

$$
h(\mathcal{O})=h(A) t(\mathcal{O}) .
$$

Moreover, for any order $\mathcal{O}^{\prime}$ in the genus of $\mathcal{O}$, we have

$$
\left|\Upsilon^{-1}\left(\llbracket \mathcal{O}^{\prime} \rrbracket\right)\right|=h(A), \quad \text { and } \quad \mathcal{N}\left(\mathcal{O}^{\prime}\right)=F^{\times} \mathcal{O}^{\prime \times} .
$$

Proof. The assumption $\mathcal{N}(\widehat{\mathcal{O}})=\widehat{F}^{\times} \widehat{\mathcal{O}}^{\times}$allows the canonical identification $\overline{\mathrm{Cl}}(\mathcal{O})=$ $\mathcal{T}(\mathcal{O})$, and hence identifications of the orbits of the $\operatorname{Pic}(A)$-action with the fibers of $\Upsilon$. In particular, we have $t(\mathcal{O})=r(\mathcal{O})$. By definition, $\widehat{\mathcal{O}}^{\prime} \simeq \widehat{\mathcal{O}}$ for any order $\mathcal{O}^{\prime}$ in the genus of $\mathcal{O}$. Hence $\mathcal{N}\left(\mathcal{O}^{\prime}\right)=D^{\times} \cap \mathcal{N}\left(\widehat{\mathcal{O}}^{\prime}\right)=\mathcal{N}_{0}\left(\mathcal{O}^{\prime}\right)$.

Suppose further that $\mathcal{O}$ is stable under the canonical involution and $h(A)$ is odd. By Corollary 2.5. $\operatorname{Pic}(A)$ acts freely on $\mathrm{Cl}(\mathcal{O})$, so we have $t(\mathcal{O})=r(\mathcal{O})=$ $h(\mathcal{O}) / h(A)$, and $\left|\Upsilon^{-1}\left(\llbracket \mathcal{O}^{\prime} \rrbracket\right)\right|=h(A)$ for each $\llbracket \mathcal{O}^{\prime} \rrbracket \in \mathcal{T}(\mathcal{O})$. The equality $\mathcal{N}\left(\mathcal{O}^{\prime}\right)=$ $F^{\times} \mathcal{O}^{\prime \times}$ follows from Lemma 2.7 . 
It is well known that the condition $\mathcal{N}(\widehat{\mathcal{O}})=\widehat{F}^{\times} \widehat{\mathcal{O}}^{\times}$holds if $D$ is unramified at all the finite places of $F$ and $\mathcal{O}$ is a maximal $O_{F}$-order (see Corollary 3.5). We provide a class of non-maximal orders (namely, $\mathbb{O}_{16}$ defined by (4.1) and (4.2) that satisfies this condition in Section 4 .

\section{PicARD GRoup aCtion For totally DEFInite QUATERnion ALGEBRAS}

Throughout this section, we assume that $F$ is a totally real field, and $D$ is a totally definite quaternion $F$-algebra. In particular, for any $\lambda \in D$ with $\lambda \notin F$, the $F$-algebra $F[\lambda]$ is a CM-extension of $F$. Let $A$ be a $\mathbb{Z}$-order in $F$, and $\mathcal{O}$ be a proper $A$-order in $D$. The main goal of this section is to derive a orbit number formula for the $\operatorname{Pic}(A)$ action on $\mathrm{Cl}(\mathcal{O})$. This also leads to a type number formula for $t(D)$ when $F$ has even degree over $\mathbb{Q}$ and $D$ is unramified at all finite places of $F$.

Notation 3.1. Let $R_{1} \subseteq R_{2}$ be an extension of commutative Noetherian rings. The canonical homomorphism between the Picard groups is denoted by

$$
i_{R_{2} / R_{1}}: \operatorname{Pic}\left(R_{1}\right) \rightarrow \operatorname{Pic}\left(R_{2}\right), \quad[\mathfrak{a}] \mapsto\left[\mathfrak{a} R_{2}\right] .
$$

If $F_{2} / F_{1}$ is a finite extension of number fields, we write $i_{F_{2} / F_{1}}$ for $i_{O_{F_{2}} / O_{F_{1}}}$. Because of Corollary 2.2, we are interested in the following set of isomorphism classes of CM proper $A$-orders:

$$
\mathscr{B}:=\left\{\llbracket B \rrbracket \mid B \text { is a } C M \text { proper } A \text {-order, and } \operatorname{ker}\left(i_{B / A}\right) \neq\{1\}\right\},
$$

where $\llbracket B \rrbracket$ denotes the $A$-isomorphism class of $B$.

Proposition 3.2. The set $\mathscr{B}$ is finite.

Proof. Let $B$ be a CM proper $A$-order with $\llbracket B \rrbracket \in \mathscr{B}$ and $K$ be its fractional field. Pick a nontrivial ideal class $[\mathfrak{a}] \in \operatorname{ker}\left(i_{B / A}\right)$ so that

$$
\mathfrak{a} B=\lambda B \quad \text { with } \quad \lambda \in K^{\times} .
$$

Necessarily $\lambda \notin F^{\times}$, otherwise $\mathfrak{a}=\lambda A$ by part (ii) of Lemma 2.1 .

There is a commutative diagram

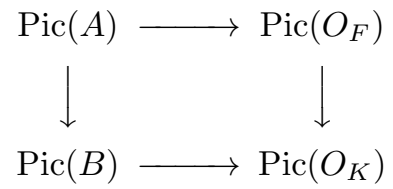

If $[\mathfrak{a}] \notin \operatorname{ker}\left(i_{O_{F} / A}\right)$, then $\left[\mathfrak{a} O_{F}\right]$ is a nontrivial ideal class in $\operatorname{ker}\left(i_{K / F}\right)$. By [3] Section 14], there are only finitely many CM-extensions $K^{\prime} / F \operatorname{such}$ that $\operatorname{ker}\left(i_{K^{\prime} / F}\right) \neq$ $\{1\}$. On the other hand, suppose that $[\mathfrak{a}] \in \operatorname{ker}\left(i_{O_{F} / A}\right)$, so that $\mathfrak{a} O_{F}=\gamma O_{F}$ for some $\gamma \in F^{\times}$. We have $\gamma O_{K}=\mathfrak{a} O_{K}=\lambda O_{K}$, and hence $\lambda=\gamma u$ for some $u \in O_{K}^{\times}$. Note that $u \notin O_{F}^{\times}$since $\lambda \notin F^{\times}$. There are only finitely many CMextensions $K^{\prime \prime} / F$ such that $\left[O_{K^{\prime \prime}}^{\times}: O_{F}^{\times}\right]>1$. Indeed, let $\boldsymbol{\mu}\left(K^{\prime \prime}\right)$ be the group of roots of unity in $K^{\prime \prime}$. There are only finitely many CM-extensions $K^{\prime \prime} / F$ such that $\boldsymbol{\mu}\left(K^{\prime \prime}\right) \neq\{ \pm 1\}$. If $\boldsymbol{\mu}\left(K^{\prime \prime}\right)=\{ \pm 1\}$, then $\left[O_{K^{\prime \prime}}^{\times}: O_{F}^{\times}\right]>1$ if and only if $K^{\prime \prime} / F$ is a CM-extension of type II (i.e. $\left[O_{K^{\prime \prime}}^{\times}: O_{F}^{\times} \boldsymbol{\mu}\left(K^{\prime \prime}\right)\right]=2$ ), and there are finitely many of these by [3, Lemma 13.3]. We conclude that the following set of CM-extensions of $F$ is finite:

$$
\mathscr{K}:=\left\{B \otimes_{A} F \mid \llbracket B \rrbracket \in \mathscr{B}\right\} .
$$


Now fix $K \in \mathscr{K}$ and a nontrivial $A$-ideal class $[\mathfrak{a}] \in \operatorname{ker}\left(i_{O_{K} / A}\right)$. Pick $\lambda^{\prime} \in K^{\times}$ such that $\mathfrak{a} O_{K}=\lambda^{\prime} O_{K}$. The CM proper $A$-orders $B \subseteq O_{K}$ such that $[\mathfrak{a}] \in$ $\operatorname{ker}\left(i_{B / A}\right)$ are characterized in Lemma 3.3 below. For each $u \in O_{K}^{\times}$with $u^{-1} \lambda^{\prime} \notin$ $F^{\times}$, we have $A \cap \frac{u \mathfrak{a}}{\lambda^{\prime}}=\{0\}$, so $A+\frac{u \mathfrak{a}}{\lambda^{\prime}}$ forms an $A$-lattice in $O_{K}$. There are only finitely many $A$-orders in $O_{K}$ containing $A \oplus \frac{u \mathfrak{a}}{\lambda^{\prime}}$. On the other hand, there is oneto-one correspondence between $O_{K}^{\times} / A^{\times}$and the set of $A$-submodules of the form $u \mathfrak{a} / \lambda^{\prime}$ in $O_{K}$. Since $K / F$ is a CM-extension, $O_{K}^{\times} / A^{\times}$is a finite group. Therefore, there are only finitely many CM proper $A$-order $B$ in $K$ with $[\mathfrak{a}] \in \operatorname{ker}\left(i_{B / A}\right)$. Since $\operatorname{ker}\left(i_{O_{K} / A}\right)$ is finite as well, the proposition follows.

Lemma 3.3. Let $K / F$ be a CM-extension with $\operatorname{ker}\left(i_{O_{K} / A}\right) \neq\{1\}$, and [a] be a nontrivial $A$-ideal class in $\operatorname{ker}\left(i_{O_{K} / A}\right)$ so that $\mathfrak{a} O_{K}=\lambda^{\prime} O_{K}$ for some $\lambda^{\prime} \in K^{\times}$. Given a $C M$ proper $A$-order $B \subseteq O_{K}$, we have $[\mathfrak{a}] \in \operatorname{ker}\left(i_{B / A}\right)$ if and only if there exists $u \in O_{K}^{\times}$such tha $u \mathfrak{a} / \lambda^{\prime} \subseteq B$. In addition, we always have $u^{-1} \lambda^{\prime} \notin F^{\times}$if such a unit $u \in O_{K}^{\times}$exists.

Proof. First, suppose that $[\mathfrak{a}] \in \operatorname{ker}\left(i_{B / A}\right)$ so that (3.2) holds. We have $\lambda^{\prime} O_{K}=$ $\mathfrak{a} O_{K}=\lambda O_{K}$, and hence $\lambda^{\prime}=u \lambda$ for some $u \in O_{K}^{\times}$. It follows that

$$
u \mathfrak{a} / \lambda^{\prime}=\mathfrak{a} / \lambda \subset B .
$$

As remarked right after (3.2),$u^{-1} \lambda^{\prime}=\lambda \notin F^{\times}$since [a] $]$is assumed to be nontrivial.

Conversely, suppose that there exists $u \in O_{K}^{\times}$such that $u \mathfrak{a} / \lambda^{\prime} \subset B$. Let $\mathfrak{b}=\frac{u \mathfrak{a}}{\lambda^{\prime}} B$, the ideal of $B$ generated by $u \mathfrak{a} / \lambda^{\prime}$. Then $\mathfrak{b} O_{K}=\frac{u \mathfrak{a}}{\lambda^{\prime}} O_{K}=O_{K}$. It follows from Lemma 2.1 that $\mathfrak{b}=B$, i.e. $\mathfrak{a} B=\left(u^{-1} \lambda^{\prime}\right) B$.

When $A$ coincides with the maximal order $O_{F}$ in $F$, we give a more precise characterization of $O_{F}$-orders $B$ in $K$ with $\operatorname{ker}\left(i_{B / O_{F}}\right) \neq\{1\}$ in Lemma 5.1.

Let $B$ be an arbitrary CM proper $A$-order with fractional field $K$, and $x \mapsto \bar{x}$ be the unique nontrivial involution of $K / F$. We define a symbol

$$
\delta(B):= \begin{cases}1 & \text { if } B=\bar{B}, \\ 0 & \text { otherwise. }\end{cases}
$$

Note that $\delta(B)=1$ for all $O_{F}$-orders $B$. If $\mathcal{O}$ is stable under the canonical involution of $D$, then so is $\mathcal{O}_{l}(I)$ for every locally principal right $\mathcal{O}$-ideal $I$, in which case $\delta\left(F(\lambda) \cap \mathcal{O}_{l}(I)\right)=1$ for every $\lambda \in D^{\times}$with $\lambda \notin F^{\times}$.

Lemma 3.4. If $B$ is a $C M$ proper $A$-order with $\delta(B)=1$, then $\left|\operatorname{ker}\left(i_{B / A}\right)\right| \leq 2$.

Proof. This result is well known when $A=O_{F}$ and $B=O_{K}$. In fact, the proof of [35. Theorem 10.3] applies, mutatis mutandis, to the current setting as well and shows that $\left|\operatorname{ker}\left(i_{B / A}\right)\right| \leq 2$.

Lemma 3.4 does not hold in general when $\delta(B)=0$. We exhibit in Example 4.4 an infinite family of pairs $(A, B)$ such that $\operatorname{ker}\left(i_{B / A}\right) \simeq \mathbb{Z} / 3 \mathbb{Z}$.

Denote by $\operatorname{Emb}(B, \mathcal{O})$ the set of optimal embeddings from $B$ into $\mathcal{O}$ :

$$
\operatorname{Emb}(B, \mathcal{O})=\left\{\varphi \in \operatorname{Hom}_{F}(K, D) \mid \varphi(K) \cap \mathcal{O}=\varphi(B)\right\} .
$$

Fix a nontrivial $A$-ideal class $[\mathfrak{a}] \in \operatorname{Pic}(A)$. We define a few subsets of $\mathscr{B}$ :

$$
\mathscr{B}(\mathcal{O})=\{\llbracket B \rrbracket \in \mathscr{B} \mid \operatorname{Emb}(B, \mathcal{O}) \neq \emptyset\},
$$

\footnotetext{
${ }^{2}$ Up to multiplication by a unit in $O_{K}^{\times}$, the $A$-submodule $\mathfrak{a} / \lambda^{\prime} \subset O_{K}$ depends only on the $A$-ideal class $[\mathfrak{a}] \in \operatorname{ker}\left(i_{O_{K} / A}\right)$ and not on the choice of $\mathfrak{a}$.
} 


$$
\begin{aligned}
\mathscr{B}_{[\mathfrak{a}]} & =\left\{\llbracket B \rrbracket \in \mathscr{B} \mid[\mathfrak{a}] \in \operatorname{ker}\left(i_{B / A}\right)\right\}, \\
\mathscr{B}_{[\mathfrak{a}]}(\mathcal{O}) & =\left\{\llbracket B \rrbracket \in \mathscr{B} \mid[\mathfrak{a}] \in \operatorname{ker}\left(i_{B / A}\right), \text { and } \operatorname{Emb}(B, \mathcal{O}) \neq \emptyset\right\} .
\end{aligned}
$$

Corollary 2.2 implies that for any ideal class $[I] \in \mathrm{Cl}(\mathcal{O})$,

$$
\operatorname{Stab}([I])=\bigcup_{\llbracket B \rrbracket \in \mathscr{B}\left(\mathcal{O}_{l}(I)\right)} \operatorname{ker}\left(i_{B / A}: \operatorname{Pic}(A) \rightarrow \operatorname{Pic}(B)\right) .
$$

Suppose that $\mathscr{B}_{[\mathfrak{a}]}\left(\mathcal{O}_{l}(I)\right) \neq \emptyset$ for some ideal class $[I] \in \mathrm{Cl}(\mathcal{O})$ so that $[\mathfrak{a}] \in$ $\operatorname{Stab}([I])$. It then follows from (2.5) that $\mathfrak{a}^{2} \widetilde{A}=\operatorname{Nr}(\lambda) \widetilde{A}$ for some $\lambda \in D^{\times}$, where $\widetilde{A}=\operatorname{Nr}_{A}(\mathcal{O})$ is the suborder of $O_{F}$ spanned over $A$ by the reduced norms of elements of $\mathcal{O}$. Note that $\operatorname{Nr}(\lambda)$ is totally positive since $D$ is totally definite over $F$. For any $\mathbb{Z}$-order $A^{\prime}$ in $F$, Let $\mathrm{Pic}^{+}\left(A^{\prime}\right)$ be the quotient of the multiplicative group of locally principal fractional $A^{\prime}$-ideals by the subgroup of principal fractional $A^{\prime}$-ideals that are generated by totally positive elements. If $A^{\prime}=O_{F}$, then $\operatorname{Pic}^{+}\left(A^{\prime}\right)$ is simply the narrow class group of $F$. We denote the subgroup of 2-torsions of $\mathrm{Pic}^{+}\left(A^{\prime}\right)$ by $\operatorname{Pic}^{+}\left(A^{\prime}\right)[2]$, and the canonical map $\operatorname{Pic}^{+}\left(A^{\prime}\right) \rightarrow \operatorname{Pic}\left(A^{\prime}\right)$ by $\theta_{A^{\prime}}$. For any order $A^{\prime}$ intermediate to $A \subseteq O_{F}$, let us define

$$
\mathfrak{C}_{2}\left(A, A^{\prime}\right):=\left\{[\mathfrak{a}] \in \operatorname{Pic}(A) \mid i_{A^{\prime} / A}([\mathfrak{a}]) \in \theta_{A^{\prime}}\left(\operatorname{Pic}^{+}\left(A^{\prime}\right)[2]\right)\right\} .
$$

If $A=O_{F}$, then necessarily $A^{\prime}=O_{F}$ as well, and we simply write $\mathfrak{C}_{2}(F)$ for $\mathfrak{C}_{2}\left(O_{F}, O_{F}\right)$, which coincides with the image of $\operatorname{Pic}^{+}\left(O_{F}\right)[2]$ in $\operatorname{Pic}\left(O_{F}\right)$. Clearly, $\mathfrak{C}_{2}(A, \widetilde{A})$ is a subgroup of $\operatorname{ker}(\operatorname{S} q)$, where $\operatorname{Sq}: \operatorname{Pic}(A) \rightarrow \operatorname{Pic}(\widetilde{A})$ is the homomorphism defined in (2.4). By our construction,

$$
[\mathfrak{a}] \notin \mathfrak{C}_{2}(A, \widetilde{A}) \quad \text { implies that } \quad[\mathfrak{a}] \notin \operatorname{Stab}([I]), \forall[I] \in \mathrm{Cl}(\mathcal{O}) \text {. }
$$

Using the norm map $\mathrm{N}_{K / F}$ for CM-extensions $K / F$, one shows similarly that $[\mathfrak{a}] \in \mathfrak{C}_{2}\left(A, O_{F}\right)$ if $\mathscr{B}_{[\mathfrak{a}]} \neq \emptyset$. We claim that this is in fact an "if and only if" condition when $A=O_{F}$. If $\mathfrak{a}$ is a non-principal fractional $O_{F}$-ideal such that $\mathfrak{a}^{2}=\tau O_{F}$ for some totally positive $\tau \in F^{\times}$, then $K=F(\sqrt{-\tau})$ is a CM-extension of $F$ with $[\mathfrak{a}] \in \operatorname{ker}\left(i_{K / F}\right)$ (cf. [3, Theorem 14.2]). Thus $\mathscr{B}_{[\mathfrak{a}]} \neq \emptyset$ if and only if $[\mathfrak{a}] \in \mathfrak{C}_{2}(F)$. Combining this with Lemma 3.4, we obtain that

$$
\mathscr{B}=\coprod_{1 \neq[\mathfrak{a}] \in \mathfrak{C}_{2}(F)} \mathscr{B}_{[\mathfrak{a}]} \quad \text { if } A=O_{F} .
$$

Therefore, $\mathscr{B}=\emptyset$ if and only if $\mathfrak{C}_{2}(F)$ is trivial.

Corollary 3.5. Let $F$ be a totally real number field of even degree over $\mathbb{Q}$, and $\mathcal{O}$ be a maximal $O_{F}$-order in the (unique up to isomorphism) totally definite quaternion $F$-algebra $D$ unramified at all finite places of $F$. The following are equivalent:

(1) the group $\mathfrak{C}_{2}(F)$ is trivial;

(2) the action of $\operatorname{Pic}\left(O_{F}\right)$ on $\mathrm{Cl}(\mathcal{O})$ is free;

(3) $t(D)=h(D) / h(F)$.

Moreover, if $h(F)$ is odd, then the statements (1)-(3) hold.

Proof. By [34, Section II.2], we have $\mathcal{N}\left(\widehat{\mathcal{O}}^{\times}\right)=\widehat{F}^{\times} \widehat{\mathcal{O}}^{\times}$in this case, and hence $t(\mathcal{O})=r(\mathcal{O}) \geq h(D) / h(F)$. The equality $r(\mathcal{O})=h(D) / h(F)$ holds if and only if the action of $\operatorname{Pic}\left(O_{F}\right)$ on $\mathrm{Cl}(\mathcal{O})$ is free, establishing the equivalence between (2) and (3).

For the equivalence between (1) and (2), it is enough to show that the latter holds if and only if $\mathscr{B}=\emptyset$. The sufficiency is clear by (3.7). As $D$ is unramified 
at all finite places, any $\mathrm{CM}$ extension $K$ of $F$ can be embedded into $D$ by the local-global principle. If $\mathscr{B} \neq \emptyset$, then there exists a CM-extension $K$ of $F$ such that $\operatorname{ker}\left(i_{K / F}\right) \neq\{1\}$. Fix an $F$-embedding $\varphi$ from such a $K$ into $D$. The image $\varphi\left(O_{K}\right)$ is contained in a maximal $O_{F}$-order $\mathcal{O}^{\prime}$. Set $I:=\mathcal{O}^{\prime} \mathcal{O}$, which is a fractional right $\mathcal{O}$ ideal with $\mathcal{O}_{l}(I)=\mathcal{O}^{\prime}$. We have $\llbracket O_{K} \rrbracket \in \mathscr{B}\left(\mathcal{O}_{l}(I)\right)$, and hence $\operatorname{ker}\left(i_{K / F}\right) \subseteq \operatorname{Stab}([I])$ by (3.7). Therefore, $\operatorname{Pic}\left(O_{F}\right)$ acts freely on $\mathrm{Cl}(\mathcal{O})$ if and only if $\mathscr{B}=\emptyset$.

It is possible that $\mathfrak{C}_{2}(F)$ is trivial despite $h(F)$ being even. Indeed, one of such examples is given by $F=\mathbb{Q}(\sqrt{34})$ (see [3, Section 12, p. 64]). We provide two applications of Corollary 3.5. First, if $F$ is a real quadratic field satisfying condition (1) of Corollary 3.5 and $D$ is a totally definite quaternion $F$-algebra unramified at all finite places of $F$, then an explicit formula for $t(D)$ of $D$ is given in [33, p. 212].

Second, recall that $\mathcal{O}^{\times} / O_{F}^{\times}$is a finite group [34, Theorem V.1.2]. For any finite group $G$, we define

$$
\begin{aligned}
h(D, G) & :=\left|\left\{[I] \in \operatorname{Cl}(\mathcal{O}) \mid \mathcal{O}_{l}(I) / A^{\times} \simeq G\right\}\right|, \\
t(D, G) & :=\left|\left\{\llbracket \mathcal{O}^{\prime} \rrbracket \in \mathcal{T}(D) \mid \mathcal{O}^{\prime} / A^{\times} \simeq G\right\}\right| .
\end{aligned}
$$

Here $h(D, G)$ does not depends on the choice of the maximal order $\mathcal{O}$, hence denoted as such. The following result is used in [19].

Corollary 3.6. Keep the assumptions of Corollary 3.5 and suppose that $\mathfrak{C}_{2}(F)$ is trivial. Then $h(D, G)=h(F) t(D, G)$ for any finite group $G$.

Proof. The assumptions guarantee that (2.11) holds in the current setting as well. The corollary follows immediately.

We return to the general setting of this section, that is, $\mathcal{O}$ being a proper $A$-order. Following [37, Section 3], we set up the following notation

$$
\begin{aligned}
w(\mathcal{O}) & :=\left[\mathcal{O}^{\times}: A^{\times}\right], & w(B) & :=\left[B^{\times}: A^{\times}\right], \\
m(B, \mathcal{O}) & :=|\operatorname{Emb}(B, \mathcal{O})|, & m\left(B, \mathcal{O}, \mathcal{O}^{\times}\right) & :=\left|\operatorname{Emb}(B, \mathcal{O}) / \mathcal{O}^{\times}\right|,
\end{aligned}
$$

where $\mathcal{O}^{\times}$acts on $\operatorname{Emb}(B, \mathcal{O})$ from the right by $\varphi \mapsto g^{-1} \varphi g$ for all $\varphi \in \operatorname{Emb}(B, \mathcal{O})$ and $g \in \mathcal{O}^{\times}$. Note that we have

$$
m(B, \mathcal{O})=m\left(B, \mathcal{O}, \mathcal{O}^{\times}\right) w(\mathcal{O}) / w(B) .
$$

For each prime $\ell \in \mathbb{N}$, let $B_{\ell}\left(\right.$ resp. $\left.\mathcal{O}_{\ell}\right)$ be the $\ell$-adic completion of $B$ (resp. $\mathcal{O}$ ). For simplicity, we put

$$
m_{\ell}(B):=m\left(B_{\ell}, \mathcal{O}_{\ell}, \mathcal{O}_{\ell}^{\times}\right)=\left|\operatorname{Emb}\left(B_{\ell}, \mathcal{O}_{\ell}\right) / \mathcal{O}_{\ell}^{\times}\right| .
$$

It is well known [34, Theorem II.3.2] that $m_{\ell}(B)=1$ for almost all prime $\ell \in \mathbb{N}$.

Theorem 3.7. Let $A$ be a $\mathbb{Z}$-order in a totally real number field $F$, and $\mathcal{O}$ be a proper $A$-order in a totally definite quaternion $F$-algebra $D$. The number of orbits of the Picard group action (1.5) can be calculated by the following formula:

$$
r(\mathcal{O})=\frac{1}{h(A)}\left(h(\mathcal{O})+\sum_{1 \neq[\mathfrak{a}] \in \mathfrak{C}_{2}(A, \widetilde{A})} \sum_{\llbracket B \rrbracket \in \mathscr{B}_{[\mathfrak{a}]}} \frac{1}{2}(2-\delta(B)) h(B) \prod_{\ell} m_{\ell}(B)\right),
$$

where $\mathfrak{C}_{2}(A, \widetilde{A})$ is the subgroup of $\operatorname{Pic}(A)$ defined in (3.8), and $\mathscr{B}_{[\mathfrak{a}]}$ is the set of isomorphism classes of CM proper A-orders defined in (3.5). Moreover, if $A=O_{F}$, 
then

$$
r(\mathcal{O})=\frac{1}{h(F)}\left(h(\mathcal{O})+\frac{1}{2} \sum_{\llbracket B \rrbracket \in \mathscr{B}} h(B) \prod_{\ell} m_{\ell}(B)\right),
$$

where $\mathscr{B}$ is the set of isomorphism classes of $C M O_{F}$-orders in (3.1) with $A=O_{F}$.

The proof of this theorem relies on Burnside's lemma [28, Theorem 2.113], which we recall briefly for the convenience of the reader.

Lemma 3.8 (Burnside's lemma). Let $G$ be a finite group acting on a finite set $X$, and $r$ be the number of orbits. For each $g \in G$, let $X^{g} \subseteq X$ be the subset of elements fixed by $g$. Then $r=\frac{1}{G} \sum_{g \in G}\left|X^{g}\right|$.

Hence we reduce the proof of Theorem 3.7 to the calculation of $\left|\mathrm{Cl}(\mathcal{O})^{[\mathfrak{a}]}\right|$ for each $[\mathfrak{a}] \in \operatorname{Pic}(A)$, where $\operatorname{Cl}(\mathcal{O})^{[\mathfrak{a}]} \subseteq \operatorname{Cl}(\mathcal{O})$ denotes the subset of locally principal right $\mathcal{O}$-ideal classes fixed by $[\mathfrak{a}]$. By $(\underline{3.9})$, if $[\mathfrak{a}] \notin \mathfrak{C}_{2}(A, \widetilde{A})$, then $\operatorname{Cl}(\mathcal{O})^{[\mathfrak{a}]}=\emptyset$.

Proposition 3.9. For each nontrivial A-ideal class $[\mathfrak{a}] \in \mathfrak{C}_{2}(A, \widetilde{A})$,

$$
\left|\mathrm{Cl}(\mathcal{O})^{[\mathfrak{a}]}\right|=\sum_{\llbracket B \rrbracket \in \mathscr{B}_{[\mathfrak{a}]}} \frac{1}{2}(2-\delta(B)) h(B) \prod_{\ell} m_{\ell}(B) .
$$

Proof. We fix a complete set of representatives $\left\{I_{j} \mid 1 \leq j \leq h:=h(\mathcal{O})\right\}$ of the ideal classes in $\mathrm{Cl}(\mathcal{O})$, and define $\mathcal{O}_{j}:=\mathcal{O}_{l}\left(I_{j}\right)$. For each $1 \leq j \leq h$, consider the following two sets

$$
X\left(\mathcal{O}_{j}\right):=\left\{\lambda \in D^{\times} \mid \mathfrak{a} \mathcal{O}_{j}=\lambda \mathcal{O}_{j}\right\}, \quad Y\left(\mathcal{O}_{j}\right):=X\left(\mathcal{O}_{j}\right) / A^{\times},
$$

where $A^{\times}$acts on $X\left(\mathcal{O}_{j}\right)$ by multiplication. Note that $X\left(\mathcal{O}_{j}\right) \cap F=\emptyset$ since $\mathfrak{a}$ is assume to be non-principal. By (2.2), we have

$$
X\left(\mathcal{O}_{j}\right) \neq \emptyset \quad \text { if and only if }\left[I_{j}\right] \in \operatorname{Cl}(\mathcal{O})^{[\mathfrak{a}]} .
$$

If $X\left(\mathcal{O}_{j}\right) \neq \emptyset$, then $\mathcal{O}_{j}^{\times}$acts simply transitively on $X\left(\mathcal{O}_{j}\right)$ from the right by multiplication. Thus

$$
\frac{\left|Y\left(\mathcal{O}_{j}\right)\right|}{w\left(\mathcal{O}_{j}\right)}= \begin{cases}1 & \text { if }\left[I_{j}\right] \in \mathrm{Cl}(\mathcal{O})^{[\mathfrak{a}]} \\ 0 & \text { otherwise. }\end{cases}
$$

We count the cardinality $\left|Y\left(\mathcal{O}_{j}\right)\right|$ in another way. By Corollary 2.2, each $\lambda \in$ $X\left(\mathcal{O}_{j}\right)$ gives rise to a $\mathrm{CM}$ proper $A$-order $B_{\lambda}:=F(\lambda) \cap \mathcal{O}_{j}$ such that $[\mathfrak{a}] \in$ $\operatorname{ker}\left(i_{B_{\lambda} / A}\right)$. In other words, the $A$-isomorphism class $\llbracket B_{\lambda} \rrbracket$ belongs to the set $\mathscr{B}_{[\mathfrak{a}]}\left(\mathcal{O}_{j}\right)$ defined in (3.6). Thus we have

$$
\begin{gathered}
X\left(\mathcal{O}_{j}\right)=\coprod_{\llbracket B \rrbracket \in \mathscr{B}_{[\mathfrak{a}]}\left(\mathcal{O}_{j}\right)} X\left(\mathcal{O}_{j}, \llbracket B \rrbracket\right), \quad \text { where } \\
X\left(\mathcal{O}_{j}, \llbracket B \rrbracket\right):=\left\{\lambda \in X\left(\mathcal{O}_{j}\right) \mid B_{\lambda} \simeq B\right\} \subseteq X\left(\mathcal{O}_{j}\right) \subset D^{\times} .
\end{gathered}
$$

Clearly, each $X\left(\mathcal{O}_{j}, \llbracket B \rrbracket\right)$ is $A^{\times}$-stable, so we set $Y\left(\mathcal{O}_{j}, \llbracket B \rrbracket\right):=X\left(\mathcal{O}_{j}, \llbracket B \rrbracket\right) / A^{\times}$. Let $K$ be the fractional field of $B$. Similar to (3.17), we define

$$
X(B):=\left\{\lambda \in K^{\times} \mid \mathfrak{a} B=\lambda B\right\}, \quad Y(B):=X(B) / A^{\times} .
$$

Then $|Y(B)|=w(B):=\left[B^{\times}: A^{\times}\right]$. 
For each $\llbracket B \rrbracket \in \mathscr{B}_{[\mathfrak{a}]}\left(\mathcal{O}_{j}\right)$, we have an $A^{\times}$-equivariant surjective map

$$
X(B) \times \operatorname{Emb}\left(B, \mathcal{O}_{j}\right) \rightarrow X\left(\mathcal{O}_{j}, \llbracket B \rrbracket\right), \quad(\lambda, \varphi) \mapsto \varphi(\lambda) .
$$

Let $\varphi$ and $\varphi^{\prime}$ be two distinct optimal embeddings of $B$ into $\mathcal{O}_{j}$. If $\varphi(B) \neq \varphi^{\prime}(B)$, then $\varphi(X(B)) \cap \varphi^{\prime}(X(B))=\emptyset$. On the other hand, $\varphi(B)=\varphi^{\prime}(B)$ if and only if

$$
\varphi^{\prime}=\bar{\varphi}, \quad B=\bar{B} .
$$

Therefore, if $\delta(B)=0$, then the map in (3.23) is bijective; otherwise it is two-to-one. It follows that from (3.13) that

$$
\frac{\left|Y\left(\mathcal{O}_{j}, \llbracket B \rrbracket\right)\right|}{w\left(\mathcal{O}_{j}\right)}=\frac{1}{2}(2-\delta(B)) \frac{w(B) m\left(B, \mathcal{O}_{j}\right)}{w\left(\mathcal{O}_{j}\right)}=\frac{1}{2}(2-\delta(B)) m\left(B, \mathcal{O}_{j}, \mathcal{O}_{j}^{\times}\right) .
$$

A priori, (3.24) holds for all $\llbracket B \rrbracket \in \mathscr{B}_{[\mathfrak{a}]}\left(\mathcal{O}_{j}\right)$, but we may extend it to all members of $\mathscr{B}_{[\mathfrak{a}]}$. Indeed, if $\llbracket B^{\prime} \rrbracket \in \mathscr{B}_{[\mathfrak{a}]}-\mathscr{B}_{[\mathfrak{a}]}\left(\mathcal{O}_{j}\right)$, then both ends of (3.24) are zero since the sets $\operatorname{Emb}\left(B^{\prime}, \mathcal{O}_{j}\right)$ and $X\left(\mathcal{O}_{j}, \llbracket B^{\prime} \rrbracket\right)$ are both empty. Similarly, the disjoint union in (3.20) can be taken to range over all $\llbracket B \rrbracket \in \mathscr{B}_{[\mathfrak{a}]}$.

By [34, Theorem 5.11, p. 92] (see also [36, Lemma 3.2] and [37, Lemma 3.2.1]),

$$
\sum_{j=1}^{h} m\left(B, \mathcal{O}_{j}, \mathcal{O}_{j}^{\times}\right)=h(B) \prod_{\ell} m_{\ell}(B) .
$$

Summing (3.24) over all $\llbracket B \rrbracket \in \mathscr{B}_{[\mathfrak{a}]}$ and $1 \leq j \leq h$, we obtain

$$
\sum_{\llbracket B \rrbracket \in \mathscr{B}_{[\mathrm{a}]}} \sum_{j=1}^{h} \frac{\left|Y\left(\mathcal{O}_{j}, \llbracket B \rrbracket\right)\right|}{w\left(\mathcal{O}_{j}\right)}=\sum_{\llbracket B \rrbracket \in \mathscr{B}_{[\mathrm{a}]}} \frac{1}{2}(2-\delta(B)) h(B) \prod_{\ell} m_{\ell}(B) .
$$

We exchange the order of summation on the left of (3.26) and apply (3.19) to get

$$
\sum_{j=1}^{h} \sum_{\llbracket B \rrbracket \in \mathscr{B}_{[\mathfrak{a}]}} \frac{\left|Y\left(\mathcal{O}_{j}, \llbracket B \rrbracket\right)\right|}{w\left(\mathcal{O}_{j}\right)}=\sum_{j=1}^{h} \frac{\left|Y\left(\mathcal{O}_{j}\right)\right|}{w\left(\mathcal{O}_{j}\right)}=\left|\mathrm{Cl}(\mathcal{O})^{[\mathfrak{a}]}\right| .
$$

This concludes the proof of the proposition.

Proof of Theorem 3.7. The general formula for $r(\mathcal{O})$ follows by combining Burnside's lemma with Proposition 3.9. Assume that $A=O_{F}$. Then $\delta(B)=1$ for all $B$. Recall that $\mathscr{B}_{[\mathfrak{a}]} \neq \emptyset$ if and only if $1 \neq[\mathfrak{a}] \in \mathfrak{C}_{2}(F)$. Thus (3.15) is a direct consequence of (3.10).

As a consequence of Theorem 3.7 we prove the following slight generalization of [32, Corollaire 1.1, p. 81], which is stated for Eichler orders.

Corollary 3.10. Let $D$ be a totally definite quaternion algebra over a totally real field $F$, and $\mathcal{O}$ be an $O_{F}$-order in $D$. Then $h(F)$ divides $2 h(\mathcal{O})$.

Proof. It is well known [3, Corollary 5.4] that $h(B) / h(F)$ is integral for any CM $O_{F}$-order $B$. So the corollary follows directly from (3.15).

Corollary 3.11. Let $F$ be a totally real number field of even degree, and $D$ be the unique totally definite quaternion $F$-algebra up to isomorphism unramified at all finite places of $F$. We have

$$
t(D)=\frac{1}{h(F)}\left(h(D)+\frac{1}{2} \sum_{\llbracket B \rrbracket \in \mathscr{B}} h(B)\right),
$$


Proof. As shown in the proof of Corollary 3.5 we have $t(D)=r(\mathcal{O})$ for any maximal $O_{F}$-order $\mathcal{O}$ in $D$. By [34, Theorem II.3.2], $m_{\ell}(B)=1$ for every prime $\ell \in \mathbb{N}$. The corollary then follows from (3.15).

Of course, (3.28) is just a special case of the general type number formula for Eichler orders [24, Theorem A] (see also [34, Corollary V.2.6]). However, it describes more concretely the CM $O_{F}$-orders $B$ with nonzero contribution to the type number. Thanks to this, we prove in Theorem 5.4 that $h(D) / h(F)$ is an integer, which is asserted by Vignéras [32, Remarque, p. 82]. In light of (3.28), it is enough to show the cardinality of the following subset of $\mathscr{B}$ is even:

$$
\mathscr{B}_{\text {odd }}:=\{\llbracket B \rrbracket \in \mathscr{B} \mid h(B) / h(F) \text { is odd }\} .
$$

We will work out the details in Section 5 .

\section{Calculation of type numbers}

Throughout this section, we fix a prime number $p$. Let $q$ be an odd power of $p$, and $\operatorname{Sp}(\sqrt{q})$ and $\mathcal{T}(\operatorname{Sp}(\sqrt{q}))$ be as in Section 1, Let $F=\mathbb{Q}(\sqrt{p}), A=\mathbb{Z}[\sqrt{p}]$, and $D=D_{p, \infty} \otimes_{\mathbb{Q}} F$ be the unique definite quaternion $F$-algebra unramified at all finite places. We may regard $D$ as the endomorphism algebra $\operatorname{End}_{\mathbb{F}_{q}}^{0}\left(X_{0}\right)$ of a member $X_{0} / \mathbb{F}_{q}$ in $\operatorname{Sp}(\sqrt{q})$.

Let $\mathbb{O}_{1}$ be a maximal $O_{F}$-order in $D$. When $p \equiv 1(\bmod 4)$, one has $A \neq O_{F}$, and $A / 2 O_{F} \cong \mathbb{F}_{2}$. In this case, we define $A$-orders $\mathbb{O}_{r}$ in $D$ for $r \in\{8,16\}$ as follows. Let $\mathbb{O}_{8}, \mathbb{O}_{16} \subset \mathbb{O}_{1}$ be the proper $A$-orders such that

$$
\begin{gathered}
\left(\mathbb{O}_{8}\right)_{2}=\left(\begin{array}{cc}
A_{2} & 2 O_{F_{2}} \\
O_{F_{2}} & O_{F_{2}}
\end{array}\right), \quad\left(\mathbb{O}_{16}\right)_{2}=\operatorname{Mat}_{2}\left(A_{2}\right), \\
\left(\mathbb{O}_{r}\right)_{\ell}=\left(\mathbb{O}_{1}\right)_{\ell} \quad \text { for } r \in\{8,16\} \text { and every prime } \ell \neq 2 .
\end{gathered}
$$

Here for any $\mathbb{Z}$-module $M$ and any prime $\ell \in \mathbb{N}$, we write $M_{\ell}$ for $M \otimes \mathbb{Z}_{\ell}$. The order $\mathbb{O}_{r}$ is of index $r$ in $\mathbb{O}_{1}$.

By [37, Theorem 6.1.2] and [39, Theorem 1.3], there is a bijection (depending on the choice of a base point in each genus)

$$
\operatorname{Sp}(\sqrt{q}) \simeq \coprod_{r} \mathrm{Cl}\left(\mathbb{O}_{r}\right)
$$

where $r \in\{1,8,16\}$ if $p \equiv 1(\bmod 4)$ and $r=1$ otherwise. If $[I]$ is an ideal class corresponding to a class $[X]$ in $\operatorname{Sp}(\sqrt{q})$ in this bijection, then $\mathcal{O}_{l}(I) \simeq \operatorname{End}(X)$. Therefore, there is a bijection $\mathcal{T}(\operatorname{Sp}(\sqrt{q})) \simeq \coprod_{r} \mathcal{T}\left(\mathbb{O}_{r}\right)$, and

$$
T(p):=|\mathcal{T}(\operatorname{Sp}(\sqrt{q}))|=\left\{\begin{array}{lll}
t\left(\mathbb{O}_{1}\right) & \text { for } p \neq 1 & (\bmod 4) ; \\
t\left(\mathbb{O}_{1}\right)+t\left(\mathbb{O}_{8}\right)+t\left(\mathbb{O}_{16}\right) & \text { for } p \equiv 1 & (\bmod 4) .
\end{array}\right.
$$

Put $\mathbb{O}_{4}:=O_{F} \mathbb{O}_{8}$, which is of index 4 in $\mathbb{O}_{1}$. It is an Eichler order of level $2 O_{F}$, and one has $\mathbb{O}_{4}=\mathbb{O}_{1} \cap w \mathbb{O}_{1} w^{-1}$, where $w=\left(\begin{array}{ll}0 & 2 \\ 1 & 0\end{array}\right)$. Since $D$ is unramified at all finite places, $\mathcal{N}\left(\widehat{\mathbb{O}}_{1}\right)=\widehat{F}^{\times} \widehat{\mathbb{O}}_{1}^{\times}$. Clearly, $\mathcal{N}\left(\widehat{\mathbb{O}}_{8}\right) \subseteq \mathcal{N}\left(\widehat{\mathbb{O}}_{4}\right)$, and $\mathcal{N}\left(\widehat{\mathbb{O}}_{16}\right) \subseteq \mathcal{N}\left(\widehat{\mathbb{O}}_{1}\right)$. Direct calculations show that $\mathcal{N}\left(\widehat{\mathbb{O}}_{8}\right)=\widehat{F}^{\times} \widehat{\mathbb{O}}_{4}^{\times}$and $\mathcal{N}\left(\widehat{\mathbb{O}}_{16}\right)=\widehat{F}^{\times} \widehat{\mathbb{O}}_{16}^{\times}$for $p \equiv 1$ (mod 4). Therefore, we have natural bijections

$$
\mathcal{T}\left(\mathbb{O}_{1}\right) \simeq \frac{\mathrm{Cl}\left(\mathbb{O}_{1}\right)}{\operatorname{Pic}\left(O_{F}\right)}, \quad \mathcal{T}\left(\mathbb{O}_{8}\right) \simeq \frac{\mathrm{Cl}\left(\mathbb{O}_{4}\right)}{\operatorname{Pic}\left(O_{F}\right)}, \quad \mathcal{T}\left(\mathbb{O}_{16}\right) \simeq \frac{\mathrm{Cl}\left(\mathbb{O}_{16}\right)}{\operatorname{Pic}(A)} .
$$


Proposition 4.1. For every prime p, we have $t\left(\mathbb{O}_{1}\right)=h\left(\mathbb{O}_{1}\right) / h(F)$. Moreover, if $p \equiv 1(\bmod 4)$, then $t\left(\mathbb{O}_{8}\right)=h\left(\mathbb{O}_{4}\right) / h(F)$ and $t\left(\mathbb{O}_{16}\right)=h\left(\mathbb{O}_{16}\right) / h(A)$.

Proof. It is known that $h(F)$ is odd for all prime $p$ (see [3, Corollary (18.4), p. 134]). By Corollary 2.5. $\mathrm{Pic}\left(O_{F}\right)$ acts freely on $\mathrm{Cl}\left(\mathbb{O}_{1}\right)$ and $\mathrm{Cl}\left(\mathbb{O}_{4}\right)$. For $r=16$, we have $\widetilde{A}=A$. On the other hand, one easily computes that $h(A)=h(F)$ if $p \equiv 1(\bmod 8)$, and $h(A)=3 h(F) / \varpi$ if $p \equiv 5(\bmod 8)$, where $\varpi:=\left[O_{F}^{\times}: A^{\times}\right]$. Particularly, $h(A)$ is odd. Then the action of $\operatorname{Pic}(A)$ on $\operatorname{Cl}\left(\mathbb{O}_{16}\right)$ is also free by Corollary 2.5 again. The proposition then follows from (4.5).

For any square free integer $d \in \mathbb{Z}$, we write $h(d)$ for the class number $h(\mathbb{Q}(\sqrt{d}))$. The discriminant of $F / \mathbb{Q}$ is denoted by $\mathfrak{d}_{F}$. We also set $K_{j}:=F(\sqrt{-j})=$ $\mathbb{Q}(\sqrt{p}, \sqrt{-j})$ for $j \in\{1,2,3\}$.

Lemma 4.2. Assume that $p \equiv 1(\bmod 4)$. Then

$$
h\left(\mathbb{O}_{4}\right)=\left\{\begin{array}{lll}
\frac{9}{2} \zeta_{F}(-1) h(F)+\frac{1}{4} h\left(K_{1}\right) & \text { for } p \equiv 1 & (\bmod 8) ; \\
\frac{5}{2} \zeta_{F}(-1) h(F)+\frac{1}{4} h\left(K_{1}\right)+\frac{2}{3} h\left(K_{3}\right) & \text { for } p \equiv 5 & (\bmod 8) .
\end{array}\right.
$$

In particular, $h\left(\mathbb{O}_{4}\right)=1$ if $p=5$.

The special value $\zeta_{F}(-1)$ of the Dedekind zeta-function $\zeta_{F}(s)$ can be calculated by Siegel's formula [40, Table 2, p. 70]. It is also known that $\zeta_{F}(-1)=\frac{1}{24} B_{2, \chi}$, where $B_{2, \chi}$ is the generalized Bernoulli number attached to the primitive quadratic Dirichlet character (of conductor $\mathfrak{d}_{F}$ ) associated to the quadratic field $F$. This can be obtained, for example, by combining [40, formula (16), p. 65] together with 35, Exercise 4.2(a)].

Proof. In this case $\mathbb{O}_{4}$ is an Eichler order of square-free level $2 O_{F}$. Let $m_{2}(B)$ be as in (3.14), with $\ell=2$ and $\mathcal{O}=\mathbb{O}_{4}$. Using the formula [34, p. 94] (cf. [37, Section 3.4]), we compute that $m_{2}\left(O_{K_{1}}\right)=1$ and $m_{2}\left(O_{K_{3}}\right)=1-\left(\frac{2}{p}\right)$, where $\left(\frac{2}{p}\right)$ is the Legendre symbol. Let $\zeta_{n}$ be a primitive $n$-th root of unity for each $n>1$. When $p=5$, we also need to compute $m_{2}\left(\mathbb{Z}\left[\zeta_{10}\right]\right)$, which is zero. Using Eichler's class number formula (see [34, Chapter V]) and the data [38, Sect. 2.8 and Prop. 3.1], we obtain a formula for $h\left(\mathbb{O}_{4}\right)$. One can also apply Vignéras's formula [32, Theorem 3.1] to the Eichler order $\mathbb{O}_{4}$.

Proof of Theorem 1.2. By [32, Theorem 3.1] (cf. 37, Proposition 6.2.10]), we have $h\left(\mathbb{O}_{1}\right)=1,2,1$ for $p=2,3,5$, respectively. Since $h(F)=h(p)=1$ for $p=2,3$ and 5 , by Proposition 4.1 we obtain

$$
t\left(\mathbb{O}_{1}\right)=1,2,1 \quad \text { for } p=2,3,5, \text { respectively. }
$$

By Lemma 4.2 and [37, Proposition 6.2.10], we have $h\left(\mathbb{O}_{4}\right)=h\left(\mathbb{O}_{16}\right)=1$ for $p=5$. Therefore,

$$
t\left(\mathbb{O}_{8}\right)=t\left(\mathbb{O}_{16}\right)=1 \quad \text { for } p=5 .
$$
have

Assume that $p \equiv 3(\bmod 4)$ and $p \geq 7$. By [37, (6.17)] and Proposition 4.1, we

$$
t\left(\mathbb{O}_{1}\right)=\frac{\zeta_{F}(-1)}{2}+\left(13-5\left(\frac{2}{p}\right)\right) \frac{h(-p)}{8}+\frac{h(-2 p)}{4}+\frac{h(-3 p)}{6} .
$$


Assume $p \equiv 1(\bmod 4)$ and $p \geq 7$. By [37, (6.16)] and Proposition 4.1], we obtain

$$
t\left(\mathbb{O}_{1}\right)=\frac{\zeta_{F}(-1)}{2}+\frac{h(-p)}{8}+\frac{h(-3 p)}{6} .
$$

By Proposition 4.1 and Lemma 4.2 , we obtain

$$
t\left(\mathbb{O}_{8}\right)=\left\{\begin{array}{lll}
\frac{9}{2} \zeta_{F}(-1)+h(-p) / 8 & \text { for } p \equiv 1 \quad(\bmod 8) ; \\
\frac{5}{2} \zeta_{F}(-1)+h(-p) / 8+h(-3 p) / 3 & \text { for } p \equiv 5 \quad(\bmod 8) .
\end{array}\right.
$$

Here we use the result of Herglotz [13] (see also [32, Chapter 3] and [38, Subsection 2.10]) to factor out $h(F)$ from $h\left(K_{1}\right)$ and $h\left(K_{3}\right)$. By the formulas for $h\left(\mathbb{O}_{16}\right)$ [37, Section 6.2.4] and Proposition 4.1, we get

$$
t\left(\mathbb{O}_{16}\right)=\left\{\begin{array}{lll}
3 \zeta_{F}(-1)+h(-p) / 4+h(-3 p) / 2 & \text { for } p \equiv 1 \quad(\bmod 8) ; \\
5 \zeta_{F}(-1)+h(-p) / 4+h(-3 p) / 6 & \text { for } p \equiv 5 \quad(\bmod 8) .
\end{array}\right.
$$

Theorem 1.2 follows from (4.4) and (4.7) - (4.12).

Remark 4.3. (1) Comparing our formulas for $h\left(\mathbb{O}_{8}\right)$ and $h\left(\mathbb{O}_{4}\right)$, we see that $h\left(\mathbb{O}_{4}\right)=h\left(\mathbb{O}_{8}\right)$ if $p \equiv 1(\bmod 8)$, or if $p \equiv 5(\bmod 8)$ and $\varpi=\left[O_{F}^{\times}: A^{\times}\right]=3$. In both cases, we have $h(A)=h(F)$, and hence

$$
\begin{aligned}
T(p) & =\frac{h\left(\mathbb{O}_{1}\right)}{h(F)}+\frac{h\left(\mathbb{O}_{4}\right)}{h(F)}+\frac{h\left(\mathbb{O}_{16}\right)}{h(A)} \\
& =\frac{h\left(\mathbb{O}_{1}\right)}{h(F)}+\frac{h\left(\mathbb{O}_{8}\right)}{h(F)}+\frac{h\left(\mathbb{O}_{16}\right)}{h(F)}=\frac{H(p)}{h(F)} .
\end{aligned}
$$

(2) Note that $\mathbb{O}_{4}$ is also defined for $p \not \equiv 1(\bmod 4)$, as an Eichler order of (non-square-free) level $2 O_{F}$. We calculate $h\left(\mathbb{O}_{4}\right)$ and obtain that $h\left(\mathbb{O}_{4}\right)=1,2$ for $p=2,3$, and for $p \geq 7$ and $p \equiv 3(\bmod 4)$,

$$
h\left(\mathbb{O}_{4}\right)=3 \zeta_{F}(-1) h(F)+\left(15-3\left(\frac{2}{p}\right)\right) \frac{h\left(K_{1}\right)}{4} .
$$

More work is needed for computing the numbers of local optimal embeddings at 2 . We omit the details as (4.14) is not used in the present paper.

Assume that $p \equiv 1(\bmod 4)$. Let us calculate $r\left(\mathbb{O}_{8}\right)$, the number of orbits for the $\operatorname{Pic}(A)$-action on $\mathrm{Cl}\left(\mathbb{O}_{8}\right)$. As mentioned above, if either $p \equiv 5(\bmod 8)$ and $\varpi=3$ or $p \equiv 1(\bmod 8)$, then we have a natural isomorphism $\operatorname{Pic}(A) \simeq \operatorname{Pic}\left(O_{F}\right)$. Since the class number of $F$ is odd, $\operatorname{Pic}(A)$ acts freely on $\mathrm{Cl}\left(\mathbb{O}_{8}\right)$ in this case by Lemma 2.3. Combining with part (1) of Remark 4.3, we obtain

$$
r\left(\mathbb{O}_{8}\right)=\frac{h\left(\mathbb{O}_{4}\right)}{h(F)}=t\left(\mathbb{O}_{8}\right) \text {. }
$$

Surprisingly, this holds for the case that $p \equiv 5(\bmod 8)$ and $\varpi=1$ as well (to be proved in Subsection 4.5). But before that, we relax the condition on $p$ a little bit and study a more general real quadratic field $F=\mathbb{Q}(\sqrt{d})$.

Example 4.4. Let $d \in \mathbb{N}$ be a square free positive integer congruent to 5 modulo 8. Whether the fundamental unit $\varepsilon$ of $F=\mathbb{Q}(\sqrt{d})$ lies in $A=\mathbb{Z}[\sqrt{d}]$ or not is a classical problem that dates back to Eisenstein [9], and there seems to be no simple criterion on $d$ for it. However, it is known [1,29] that the number of $d$ for each case is infinite. By [1, Section 3], the kernel of $i_{O_{F} / A}: \operatorname{Pic}(A) \rightarrow \operatorname{Pic}\left(O_{F}\right)$ is generated by 
the locally principal $A$-ideal class $[\mathfrak{a}]$ represented by $\mathfrak{a}=4 \mathbb{Z}+(1+\sqrt{d}) \mathbb{Z}$. Moreover, $\mathfrak{a}^{3}=8 A$, and $\mathfrak{a}$ is non-principal if and only if $\varepsilon \in A$.

Let $K=\mathbb{Q}(\sqrt{d}, \sqrt{-3})$, and $B_{3,2}=A\left[\sqrt{-3}, \frac{1+\sqrt{d}}{2} \zeta_{6}\right]$ with $\zeta_{6}=(1+\sqrt{-3}) / 2$. One calculates that

$$
B_{3,2}=\mathbb{Z}+\mathbb{Z} \sqrt{d}+\mathbb{Z} \frac{\sqrt{d}+\sqrt{-3}}{2}+\mathbb{Z} \frac{(1+\sqrt{d})(1+\sqrt{-3})}{4} \subset O_{K} .
$$

In particular, $B_{3,2}$ is a $\mathrm{CM}$ proper $A$-order with $\left[O_{K}: B_{3,2}\right]=2$ and $\delta\left(B_{3,2}\right)=0$. We have $2 O_{K} \subset B$, and $B / 2 O_{K}=\mathbb{F}_{4} \oplus \mathbb{F}_{2} \subset \mathbb{F}_{4} \oplus \mathbb{F}_{4}=O_{K} / 2 O_{K}$ (cf. [38, Section 4.8]). Note that $\zeta_{6} \notin B_{3,2}$, otherwise $B_{3,2}=O_{K}$ by [20, Exercise II.42(d), p. 51]. Thus, we have

$$
3=\left[\left(O_{K} / 2 O_{K}\right)^{\times}:\left(B_{3,2} / 2 O_{K}\right)^{\times}\right] \geq\left[O_{K}^{\times}: B_{3,2}^{\times}\right] \geq 3,
$$

and hence $\left[O_{K}^{\times}: B_{3,2}^{\times}\right]=3$. It follows from [21, Theorem I.12.12] that $h\left(B_{3,2}\right)=$ $h\left(O_{K}\right)$, and the canonical map $\operatorname{Pic}\left(B_{3,2}\right) \rightarrow \operatorname{Pic}\left(O_{K}\right)$ is an isomorphism. Therefore, $\operatorname{ker}\left(i_{B_{3,2} / A}\right)=\operatorname{ker}\left(i_{O_{K} / A}\right) \supseteq \operatorname{ker}\left(i_{O_{F} / A}\right)=\langle[\mathfrak{a}]\rangle$.

Now assume that $3 \nmid d$, and $\varepsilon \in A$ so that $\mathfrak{a}$ is non-principal. Then $K / F$ is ramified at every place of $F$ above 3 . Hence $O_{K}^{\times}=O_{F}^{\times} \boldsymbol{\mu}(K)$ and $i_{K / F}: \operatorname{Pic}\left(O_{F}\right) \rightarrow$ $\operatorname{Pic}\left(O_{K}\right)$ is an embedding by [3, Lemma 13.5]. Therefore, we have

$$
\operatorname{ker}\left(i_{B_{3,2} / A}\right)=\langle[\mathfrak{a}]\rangle \simeq \mathbb{Z} / 3 \mathbb{Z}
$$

It is known [1, Theorem 4.1] that there are infinitely many square free $d$ of the form $4 n^{2}+1$ with an odd $n>3$ such that $\varepsilon \in A$. In particular, there are infinitely many $d$ satisfying our assumptions.

4.5. We return to the case that $F=\mathbb{Q}(\sqrt{p})$ with $p \equiv 5(\bmod 8)$. Assume that $\varepsilon \in A=\mathbb{Z}[\sqrt{p}]$ so that $\operatorname{ker}\left(i_{O_{F} / A}\right)=\langle[\mathfrak{a}]\rangle \simeq \mathbb{Z} / 3 \mathbb{Z}$, with $\mathfrak{a}=4 \mathbb{Z}+(1+\sqrt{p}) \mathbb{Z}$ as in Example 4.4. In order to compute $r\left(\mathbb{O}_{8}\right)$, we list all CM proper $A$-orders $B$ with $\operatorname{ker}\left(i_{B / A}\right)$ nontrivial. Let $K$ be the fractional field of $B$. Since $h(F)$ is odd, the morphism $i_{K / F}: \operatorname{Pic}\left(O_{F}\right) \rightarrow \operatorname{Pic}\left(O_{K}\right)$ is injective, and hence $\operatorname{ker}\left(i_{B / A}\right) \subseteq$ $\operatorname{ker}\left(i_{O_{F} / A}\right)$. By the proof of Proposition [3.2, necessarily $\left[O_{K}^{\times}: O_{F}^{\times}\right]>1$, and there exists a unit $u \in O_{K}^{\times}$such that $u \notin O_{F}^{\times}$and $u \mathfrak{a} / 2 \subseteq B$. According to [38, Subsection 2.8], $K$ coincides with either $F(\sqrt{-1})$ or $F(\sqrt{-3})$, and $O_{K}^{\times}=O_{F}^{\times} \boldsymbol{\mu}(K)$ in both cases. By the assumption, $O_{F}^{\times}=A^{\times}$.

First, suppose that $K=F(\sqrt{-1})$. Then $O_{K}^{\times} / A^{\times}$is a cyclic group of order 2 generated by the coset of $\sqrt{-1}$. Note that $A[\mathfrak{a} \sqrt{-1} / 2] \supseteq O_{F}$, because

$$
\left(\frac{(1+\sqrt{p}) \sqrt{-1}}{2}\right)^{2}=-\left(\frac{p-1}{4}+\frac{1+\sqrt{p}}{2}\right) .
$$

Hence there is no CM proper $A$-order $B$ in $F(\sqrt{-1})$ with $\operatorname{ker}\left(i_{B / A}\right)$ nontrivial.

Second, suppose that $K=F(\sqrt{-3})$. Then $O_{K}^{\times} / A^{\times}$is a cyclic group of order 3 generated by the coset of $\zeta_{6}$. We have $A\left[\mathfrak{a} \zeta_{6} / 2\right]=B_{3,2}$ in (4.16), and $A\left[\mathfrak{a} \zeta_{6}^{-1} / 2\right]=$ $\bar{B}_{3,2}$, the complex conjugate of $B_{3,2}$.

Therefore, up to isomorphism, $B=B_{3,2}$ is the unique CM proper $A$-order with $\operatorname{ker}\left(i_{B / A}\right)$ nontrivial. The same proof as that in [37, Subsection 6.2.5] shows that $m_{2}\left(B_{3,2}\right)=1$ for $\mathcal{O}=\mathbb{O}_{8}$. It follows from Theorem 3.7 that

$$
r\left(\mathbb{O}_{8}\right)=\frac{1}{3 h(F)}\left(h\left(\mathbb{O}_{8}\right)+2 h\left(B_{3,2}\right)\right)=\frac{5}{2} \zeta_{F}(-1)+\frac{1}{8} h(-p)+\frac{1}{3} h(-3 p) .
$$

Comparing with (4.11), we find that (4.15) holds in the current setting as well. 


\section{Integrality of $h(D) / h(F)$ When $\mathfrak{d}(D)=O_{F}$}

Let $F$ be a totally real number field, and $D$ be a totally definite quaternion algebra over $F$. The goal of this section is two-fold: first, we characterize all the $\mathrm{CM} O_{F}$-orders $B$ for which the kernel of $i_{B / O_{F}}: \operatorname{Pic}\left(O_{F}\right) \rightarrow \operatorname{Pic}(B)$ is nontrivial; second, we show that $h(D) / h(F)$ is integral when $[F: \mathbb{Q}]$ is even and $D$ is unramified at all finite places of $F$ (i.e. the reduced discriminant $\mathfrak{d}(D)=O_{F}$ ).

For simplicity, we identify $F$ with a subfield of $\mathbb{C}$, and consider $\mathrm{CM}$ extensions of $F$ as subfields of $\mathbb{C}$ as well. This way two $\mathrm{CM} O_{F}$-orders are isomorphic if and only if they are the same. The set $\mathscr{B}$ defined in (3.1) with $A=O_{F}$ becomes to

$$
\mathscr{B}=\left\{B \mid B \text { is a } \mathrm{CM} O_{F} \text {-order, and } \operatorname{ker}\left(i_{B / O_{F}}\right) \neq\{1\}\right\} .
$$

By Lemma 3.4, $\operatorname{ker}\left(i_{B / O_{F}}\right) \simeq \mathbb{Z} / 2 \mathbb{Z}$ for every $B \in \mathscr{B}$. In particular, if $h(F)$ is odd, then $\mathscr{B}=\emptyset$ and $h(D) / h(F)$ is an integer by Corollary 3.5. So we shall focus on the case where $h(F)$ is even. The finitely many $\mathrm{CM}$ extensions $K / F$ with $\operatorname{ker}\left(i_{K / F}\right)$ nontrivial are classified in [3, Section 14]. It remains to characterize all $O_{F}$-orders $B \subseteq O_{K}$ with $\operatorname{ker}\left(i_{B / O_{F}}\right)=\operatorname{ker}\left(i_{K / F}\right)$ for each such $K$.

Lemma 5.1. Let $K / F$ be a CM-extension with $\operatorname{ker}\left(i_{K / F}\right) \neq\{1\}$, and let $[\mathfrak{a}] \in$ $\operatorname{ker}\left(i_{K / F}\right)$ be the unique nontrivial ideal class so that $\mathfrak{a} O_{K}=\lambda O_{K}$ for some $\lambda \in K^{\times}$.

(i) Suppose that $K \neq F(\sqrt{-1})$. Then $[\mathfrak{a}] \in \operatorname{ker}\left(i_{B / O_{F}}\right)$ if and only if $B$ contains the $O_{F}$-order $B_{0}:=O_{F} \oplus \mathfrak{I}$, where $\mathfrak{I}$ denotes the purely imaginary $O_{F}$-submodule $\left\{z \in O_{K} \mid \bar{z}=-z\right\}$ of $O_{K}$.

(ii) Suppose that $K=F(\sqrt{-1})$. Let $n=\max \left\{m \in \mathbb{N} \mid \mathbb{Q}\left(\zeta_{2^{m}}\right) \subseteq K\right\}$, where $\zeta_{2^{m}}$ denotes a primitive $2^{m}$-th root of unity. Put $\eta=\zeta_{2^{n}}$, and $\mathfrak{I}_{\eta}=\{z \in$ $\left.O_{K} \mid \bar{z}=\eta z\right\}$. Then $[\mathfrak{a}] \in \operatorname{ker}\left(i_{B / O_{F}}\right)$ if and only if $B$ contains the $O_{F}$-order $B_{0}:=O_{F} \oplus \mathfrak{I}_{\eta}$. Moreover, $\langle\eta\rangle \subseteq B_{0}^{\times}$, so $B_{0}$ does not depend on the choice of $\zeta_{2^{n}}$.

Proof. By Lemma 3.3, $[\mathfrak{a}] \in \operatorname{ker}\left(i_{B / A}\right)$ if and only if there exits $u \in O_{K}^{\times}$such that $u \mathfrak{a} / \lambda \subset B$. Let $\boldsymbol{\mu}(K)$ be the group of roots of unity in $K$. Because $\operatorname{ker}\left(i_{K / F}\right)$ is nontrivial, it follows from [3, Section 13, p. 68] that the CM-extension $K / F$ is of type I, i.e. $O_{K}^{\times}=O_{F}^{\times} \boldsymbol{\mu}(K)$. Write $u=v \xi$ with $v \in O_{F}^{\times}$and $\xi \in \boldsymbol{\mu}(K)$. Then $\xi \mathfrak{a} / \lambda=u \mathfrak{a} / \lambda \subset B$. Therefore, $[\mathfrak{a}] \in \operatorname{ker}\left(i_{B / O_{F}}\right)$ if and only if there exists a root of unity $\xi \in \boldsymbol{\mu}(K)$ such that $\xi \mathfrak{a} / \lambda \subset B$.

(i) Now suppose that $K \neq F(\sqrt{-1})$. By [3, Section 14], replacing $\lambda$ by $\lambda u^{\prime}$ for some $u^{\prime} \in O_{K}^{\times}$, we may assume that $\bar{\lambda}=-\lambda$ and $\mathfrak{a}^{2}=-\lambda^{2} O_{F}$. Clearly, $\mathfrak{a} / \lambda \subseteq \mathfrak{I}$, and $B_{0}:=O_{F} \oplus \mathfrak{I}$ is an $O_{F}$-suborder of $O_{K}$. By Lemma 3.3. [a] $\in \operatorname{ker}\left(i_{B / O_{F}}\right)$ for any $O_{F}$-order $B \supseteq B_{0}$.

Now we prove the other direction. We first claim that $\mathfrak{a} / \lambda=\mathfrak{I}$. Since both sides are invertible $O_{F}$-modules, there exists a nonzero $O_{F}$-ideal $\mathfrak{c} \subseteq O_{F}$ such that $\mathfrak{a} / \lambda=\mathfrak{c} \mathfrak{I}$. Then $O_{K}=\frac{\mathfrak{a}}{\lambda} O_{K}=\mathfrak{c} \mathfrak{I} O_{K} \subseteq \mathfrak{c} O_{K}$. It follows that $\mathfrak{c} O_{K}=O_{K}$, and hence $\mathfrak{c}=O_{F}$ by Lemma 2.1 .

Let $B$ be an $O_{F}$-suborder of $O_{K}$ such that $\xi \mathfrak{a} / \lambda \subseteq B$ for some $\xi \in \boldsymbol{\mu}(K)$. Replacing $\xi$ by $-\xi$ if necessary, we may assume that $\xi$ has odd order. Then $\xi^{2} \in$ $\xi^{2} O_{F}=(\xi \mathfrak{a} / \lambda)^{2} \subseteq B$, and hence $\langle\xi\rangle \subset B$. It follows that $\mathfrak{I}=\mathfrak{a} / \lambda \subseteq B$, and hence $B \supseteq B_{0}$.

(ii) Suppose that $K=F(\sqrt{-1})$. Note that both $\eta$ and $\bar{\eta}$ belong to $B_{0}$ as $1+\bar{\eta} \in \mathfrak{I}_{\eta}$ and $\eta+\bar{\eta} \in O_{F}$. If both $x$ and $y$ are elements of $\mathfrak{I}_{\eta}$, then $x y \in O_{F} \bar{\eta}$ as $\overline{x y}=(x y \eta) \cdot \eta$ and $\overline{x y \eta}=x y \eta$. Thus $B_{0}$ is an $O_{F}$-suborder of $O_{K}$, so $\langle\eta\rangle \subset B_{0}^{\times}$. 
By [3, Corollary 14.7] and the Remark on [3, p. 88], the unique nontrivial ideal class in $\operatorname{ker}\left(\operatorname{Pic}\left(O_{F}\right) \rightarrow \operatorname{Pic}\left(O_{K}\right)\right)$ is represented by an $O_{F}$-ideal $\mathfrak{a}$ such that $\mathfrak{a}^{2}=$ $(2+\eta+\bar{\eta}) O_{F}$ and $\mathfrak{a} O_{K}=(1+\eta) O_{K}$. We have $\mathfrak{a} /(1+\eta) \subseteq \mathfrak{I}_{\eta} \subset B_{0}$. By Lemma 3.3 again, $[\mathfrak{a}] \in \operatorname{ker}\left(i_{B / O_{F}}\right)$ for any order $B \supseteq B_{0}$.

Conversely, let $B \subseteq O_{K}$ be an $O_{F}$-suborder such that $\xi \mathfrak{a} /(1+\eta) \subseteq B$ for some $\xi \in \boldsymbol{\mu}(K)$. The same proof as that in (i) shows that $\mathfrak{a} /(1+\eta)=\mathfrak{I}_{\eta}$. Note that $2+\eta+\bar{\eta} \in \mathfrak{a}^{2}$. We have

$$
\xi^{2} \bar{\eta}=\frac{\xi^{2}(2+\eta+\bar{\eta})}{(1+\eta)^{2}} \in\left(\frac{\xi \mathfrak{a}}{1+\eta}\right)^{2} \subseteq B
$$

Write $\xi=\xi^{\prime} \eta^{r}$ such that $s:=\operatorname{ord}\left(\xi^{\prime}\right)$ is odd. Then

$$
\langle\eta\rangle=\left\langle\eta^{s(2 r-1)}\right\rangle=\left\langle\left(\xi^{2} \bar{\eta}\right)^{s}\right\rangle \subseteq\left\langle\xi^{2} \bar{\eta}\right\rangle \subseteq B^{\times} .
$$

It follows from (5.1) that $\xi^{\prime 2} \in B$, and hence $\xi^{\prime} \in B$ as well. We conclude that $\xi \in B$, and $\mathfrak{I}_{\eta}=\mathfrak{a} /(1+\eta) \subseteq B$. This completes the proof of the lemma.

Corollary 5.2. Keep the notation and assumptions of Lemma 5.1. The index $\left[O_{K}^{\times}: B^{\times}\right]$is odd for any $O_{F}$-order $B \subseteq O_{K}$ with $[\mathfrak{a}] \in \operatorname{ker}\left(i_{B / O_{F}}\right)$. If $K \neq F(\sqrt{-1})$, then $2 O_{K} \subseteq B$; if $K=F(\sqrt{-1})$, then $(2-\eta-\bar{\eta}) O_{K} \subseteq B$.

Proof. It is enough to prove the corollary for the case $B=B_{0}$. As pointed in the proof of Lemma 5.1 we have $O_{K}^{\times}=O_{F}^{\times} \boldsymbol{\mu}(K)$. The 2-primary subgroup of $\boldsymbol{\mu}(K)$ is contained in $B_{0}^{\times}$, so $\left[O_{K}^{\times}: B_{0}^{\times}\right]$is odd. Clearly, $2 O_{K} \subseteq B_{0}$ if $K \neq F(\sqrt{-1})$. Suppose that $K=F(\sqrt{-1})$. For any $\alpha \in O_{K}$, write $\alpha=a+b$ with $a \in F$ and $b \in \mathfrak{I}_{\eta} \otimes_{O_{F}} F \subseteq K$. We then have

$$
a=\frac{-\eta \alpha+\bar{\alpha}}{1-\eta}, \quad b=\frac{\alpha-\bar{\alpha}}{1-\eta} .
$$

Both $(2-\eta-\bar{\eta}) a$ and $(2-\eta-\bar{\eta}) b$ are integral, and the corollary follows.

Now assume that $h(F)$ is even and consider the following set in (3.29):

$$
\mathscr{B}_{\text {odd }}=\{B \in \mathscr{B} \mid h(B) / h(F) \text { is odd }\} \subseteq \mathscr{B} .
$$

Our goal is to show that $\left|\mathscr{B}_{\text {oddd }}\right|$ is even. Clearly, if $B \in \mathscr{B}_{\text {odd }}$, then $O_{K} \in \mathscr{B}_{\text {odd }}$ as well with $K$ being the fractional field of $B$. By a theorem of Kummer (cf. [3, Theorem 13.14]), if $h(F)$ is even and $h(K) / h(F)$ is odd, then $\operatorname{ker}\left(i_{K / F}\right)$ is nontrivial. The CM extensions $K / F$ with odd relative class number $h(K) / h(F)$ are classified in [3, Section 16]. We fix such a $K$ and characterize all the $O_{F}$-suborders $B \subseteq O_{K}$ that lie in $\mathscr{B}_{\text {odd }}$.

Proposition 5.3. Let $K / F$ be a CM-extension with $h(F)$ even and the relative class number $h(K) / h(F)$ odd. Denote by $\mathfrak{f}_{\diamond}$ the product of all dyadic prime $O_{F}-$ ideals that are unramified in $K$. An $O_{F}$-order $B \subseteq O_{K}$ has odd relative class number $h(B) / h(F)$ if and only if $B$ contains the $O_{F}$-order $B_{\diamond}:=O_{F}+\mathfrak{f}_{\diamond} O_{K}$. Moreover, $B_{\diamond}=O_{K}$ if and only if both of the following conditions hold:

- $F$ has a single dyadic prime, and

- it is ramified in $K / F$.

Proof. Since $h(F)$ is assumed to be even and $h(K) / h(F)$ is odd, we know by [3, Section 16] that the 2-primary subgroup $\operatorname{Pic}\left(O_{F}\right)\left[2^{\infty}\right]$ of $\operatorname{Pic}\left(O_{F}\right)$ is cyclic, and

$$
\operatorname{ker}\left(i_{K / F}: \operatorname{Pic}\left(O_{F}\right) \rightarrow \operatorname{Pic}\left(O_{K}\right)\right)=\operatorname{Pic}\left(O_{F}\right)[2] \simeq \mathbb{Z} / 2 \mathbb{Z} \text {. }
$$


Let $[\mathfrak{a}] \in \operatorname{ker}\left(i_{K / F}\right)$ be the unique nontrivial ideal class as in Lemma 5.1. Then $i_{B / O_{F}}([\mathfrak{a}]) \in \operatorname{ker}\left(\operatorname{Pic}(B) \rightarrow \operatorname{Pic}\left(O_{K}\right)\right)$ for any $O_{F}$-order $B \subseteq O_{K}$. If $h(B) / h(F)$ is odd, then $h(B) / h(K)$ is odd and $[\mathfrak{a}] \in \operatorname{ker}\left(i_{B / O_{F}}\right)$, and hence $B \supseteq B_{0}$ by Lemma 5.1 . By Corollary [5.2, for such an order $B$, its conductor $\mathfrak{f}(B) \subseteq O_{F}$ is a product of dyadic primes of $O_{F}$, and $\left[O_{K}^{\times}: B^{\times}\right]$is odd.

The class number of an $O_{F}$-order $B \subseteq O_{K}$ is given by [32, p. 75]

$$
h(B)=\frac{h(K) \mathrm{N}_{F / \mathbb{Q}}(\mathfrak{f}(B))}{\left[O_{K}^{\times}: B^{\times}\right]} \prod_{\mathfrak{p} \mid \mathfrak{f}(B)}\left(1-\frac{\left(\frac{K}{\mathfrak{p}}\right)}{\mathrm{N}_{F / \mathbb{Q}}(\mathfrak{p})}\right),
$$

where $\left(\frac{K}{\mathfrak{p}}\right)$ is the Artin's symbol [34, p. 94]. More explicitly,

$$
\left(\frac{K}{\mathfrak{p}}\right)= \begin{cases}1 & \text { if } \mathfrak{p} \text { splits in } K \\ 0 & \text { if } \mathfrak{p} \text { ramifies in } K \\ -1 & \text { if } \mathfrak{p} \text { is inert in } K\end{cases}
$$

It follows that $h(B) / h(F)$ is odd if and only if

(1) $\mathfrak{f}(B)$ divides $\mathfrak{f}_{0}:=\mathfrak{f}\left(B_{0}\right)$ and is square-free; and

(2) every prime divisor $\mathfrak{p}$ of $\mathfrak{f}(B)$ is unramified in $K$.

We claim that $\mathfrak{f}_{0}$ is divisible by every dyadic prime $\mathfrak{p}$ of $F$ that is unramified in $K$. It is enough to prove it locally, so we use a subscript $\mathfrak{p}$ to indicate completion at $\mathfrak{p}$. For example, $F_{\mathfrak{p}}$ denotes the $\mathfrak{p}$-adic completion of $F$.

First, suppose that $K=F(\sqrt{-1})$. Let $\mathfrak{p}$ be a dyadic prime of $F$, and $\nu_{\mathfrak{p}}$ be its associated valuation. By [22, 63:3], $K / F$ is unramified at $\mathfrak{p}$ if and only if there exists a unit $u \in O_{F_{\mathfrak{p}}}^{\times}$such that $-1 \equiv u^{2}\left(\bmod 4 O_{F_{\mathfrak{p}}}\right)$. Assume that this is the case. Then $O_{K_{\mathfrak{p}}}=O_{F_{\mathfrak{p}}}+O_{F_{\mathfrak{p}}}(u+\sqrt{-1}) / 2$. Given $m \in \mathbb{N}$, we have $\mathfrak{p}^{m} O_{K_{\mathfrak{p}}} \subset\left(B_{0}\right)_{\mathfrak{p}}$ if and only if $\mathfrak{p}^{m}(u+\sqrt{-1}) / 2 \subset\left(B_{0}\right)_{\mathfrak{p}}$. Write $(u+\sqrt{-1}) / 2=a+b$ with $a \in F_{\mathfrak{p}}$ and $b \in \mathfrak{I}_{\eta} \otimes_{O_{F}} F_{\mathfrak{p}}$. Then by (5.2),

$$
a=\frac{u}{2}-\frac{(1+\eta) \sqrt{-1}}{2(1-\eta)}, \quad b=\frac{\sqrt{-1}}{1-\eta} .
$$

We obtain that

$$
\nu_{\mathfrak{p}}\left(\mathfrak{f}_{0}\right)=\frac{1}{2} \nu_{\mathfrak{p}}\left(\mathrm{N}_{K / F}(1-\eta)\right)=\frac{1}{2} \nu_{\mathfrak{p}}(2-\eta-\bar{\eta})>0 .
$$

Next, suppose that $K \neq F(\sqrt{-1})$. By [3] p. 82], $K=F(\sqrt{-\varsigma})$ for some totally positive element $\varsigma \in F$ with $\nu_{\mathfrak{q}}(\varsigma) \equiv 0(\bmod 2)$ for every finite prime $\mathfrak{q}$ of $F$. Thus for every $\mathfrak{q}$, there exists a unit $u \in O_{F_{\mathfrak{q}}}^{\times}$such that $K_{\mathfrak{q}}=F(\sqrt{-u})$. Let $\mathfrak{p}$ be a dyadic prime of $F$ that is unramified in $K$. Another application of [22, 63:3] as above shows that

$$
\nu_{\mathfrak{p}}\left(\mathfrak{f}_{0}\right)=\nu_{\mathfrak{p}}(2)>0 .
$$

This conclude the verification of the claim and proves the first part of the proposition.

For the last part of the proposition, recall that by [3, Theorem 16.1], one of the following holds for $K / F$ :

- exactly one finite prime of $F$ ramifies in $K / F$ and it is dyadic; or

- no finite prime of $F$ ramifies in $K / F$. 
If $\mathfrak{f}_{\diamond}=O_{F}$, or equivalently, every dyadic prime of $F$ ramifies in $K$, then $F$ has a single dyadic prime and it ramifies in $K / F$. The converse is obvious.

As mentioned before, the following theorem is asserted by Vignéras [32, Remarque, p. 82].

Theorem 5.4. Let $F$ be a totally real number field of even degree over $\mathbb{Q}$, and $D$ be the totally definite quaternion $F$-algebra unramified at all the finite places of $F$. Then $h(D) / h(F)$ is integral.

Proof. The theorem follows from Corollary 2.5 if $h(F)$ is odd. Suppose that $h(F)$ is even. We show that the cardinality of the set $\mathscr{B}_{\text {odd }}$ in (5.3) is even. If $\operatorname{Pic}\left(O_{F}\right)[2] \not \mathbb{Z} / 2 \mathbb{Z}$, then $\mathscr{B}_{\text {odd }}=\emptyset$ by the proof of Proposition 5.3. Suppose further that $\operatorname{Pic}\left(O_{F}\right)[2] \simeq \mathbb{Z} / 2 \mathbb{Z}$, and $K / F$ is a CM-extension with odd relative class number $h(K) / h(F)$. Let $\omega\left(\mathfrak{f}_{\diamond}\right)$ be the number of prime factors of $\mathfrak{f}_{\diamond}$. Then

$$
\left|\left\{B \mid B_{\diamond} \subseteq B \subseteq O_{K}\right\}\right|=2^{\omega\left(\mathfrak{f}_{\diamond}\right)} .
$$

If $B_{\diamond} \neq O_{K}$, then $\omega\left(\mathfrak{f}_{\diamond}\right)>1$ and the right hand side of (5.8) is even. If $B_{\diamond}=O_{K}$, then $F$ has a single dyadic prime $\mathfrak{p}$, and it ramifies in $K$. By [3, Theorem 16.1] and [3, Corollary 16.2], $F$ admits exactly two CM-extensions with odd relative class numbers, and $\mathfrak{p}$ ramifies in both of them. It follows that $\left|\mathscr{B}_{\text {odd }}\right|=2$ in this case. Therefore, $\left|\mathscr{B}_{\text {odd }}\right|$ is even in all cases, as claimed. Now the theorem follows from Corollary 3.11 .

\section{ACKNOWLEDGMENTS}

The second named author is grateful to Paul Ponomarev for answering his questions on type numbers. The manuscript was prepared during the authors' visit at Münster University. They thank Urs Hartl and the institution for warm hospitality and excellent research environment. J. Xue is partially supported by the 1000-plan program for young talents and Natural Science Foundation grant \#11601395 of PRC. Yu is partially supported by the MoST grants 104-2115-M-001-001MY3 and 107-2115-M-001-001-MY2.

\section{REFERENCES}

[1] Roger C. Alperin. Remarks on a problem of Eisenstein. JP J. Algebra Number Theory Appl., $7(1): 97-102,2007$.

[2] Robert J. Bond. Capitulation in abelian extensions of number fields. Acta Arith., 179(3):201$232,2017$.

[3] P. E. Conner and J. Hurrelbrink. Class number parity, volume 8 of Series in Pure Mathematics. World Scientific Publishing Co., Singapore, 1988.

[4] Charles W. Curtis and Irving Reiner. Methods of representation theory. Vol. I. Wiley Classics Library. John Wiley \& Sons, Inc., New York, 1990. With applications to finite groups and orders, Reprint of the 1981 original, A Wiley-Interscience Publication.

[5] Max Deuring. Die Anzahl der Typen von Maximalordnungen einer definiten Quaternionenalgebra mit primer Grundzahl. Jber. Deutsch. Math. Verein., 54:24-41, 1950.

[6] M. Eichler. Über die Idealklassenzahl total definiter Quaternionenalgebren. Math. Z., 43(1):102-109, 1938.

[7] Martin Eichler. Zur Zahlentheorie der Quaternionen-Algebren. J. Reine Angew. Math., 195:127-151 (1956), 1955.

[8] David Eisenbud. Commutative algebra with a view toward algebraic geometry, volume 150 of Graduate Texts in Mathematics. Springer-Verlag, New York, 1995.

[9] G. Eisenstein. Aufgaben. J. Reine Angew. Math., 27:86-88, 1844. 
[10] Ki-ichiro Hashimoto and Tomoyoshi Ibukiyama. On class numbers of positive definite binary quaternion Hermitian forms. J. Fac. Sci. Univ. Tokyo Sect. IA Math., 27(3):549-601, 1980.

[11] Ki-ichiro Hashimoto and Tomoyoshi Ibukiyama. On class numbers of positive definite binary quaternion Hermitian forms. II. J. Fac. Sci. Univ. Tokyo Sect. IA Math., 28(3):695-699 (1982), 1981.

[12] Ki-ichiro Hashimoto and Tomoyoshi Ibukiyama. On class numbers of positive definite binary quaternion Hermitian forms. III. J. Fac. Sci. Univ. Tokyo Sect. IA Math., 30(2):393-401, 1983.

[13] G. Herglotz. Über einen Dirichletschen Satz. Math. Z., 12(1):255-261, 1922.

[14] Tomoyoshi Ibukiyama, Toshiyuki Katsura, and Frans Oort. Supersingular curves of genus two and class numbers. Compositio Math., 57(2):127-152, 1986.

[15] Kenkichi Iwasawa. A note on capitulation problem for number fields. Proc. Japan Acad. Ser. A Math. Sci., 65(2):59-61, 1989.

[16] H. Jacobinski. Genera and decompositions of lattices over orders. Acta Math., 121:1-29, 1968.

[17] Toshiyuki Katsura and Frans Oort. Families of supersingular abelian surfaces. Compositio Math., 62(2):107-167, 1987.

[18] Otto Körner. Traces of Eichler-Brandt matrices and type numbers of quaternion orders. Proc. Indian Acad. Sci. Math. Sci., 97(1-3):189-199 (1988), 1987.

[19] Q. Li, J. Xue, and C.-F. Yu. Unit groups of maximal orders in totally definite quaternion algebras over real quadratic fields. ArXiv e-prints, July 2018, arXiv:1807.04736

[20] Daniel A. Marcus. Number fields. Springer-Verlag, New York, 1977. Universitext.

[21] Jürgen Neukirch. Algebraic number theory, volume 322 of Grundlehren der Mathematischen Wissenschaften [Fundamental Principles of Mathematical Sciences]. Springer-Verlag, Berlin, 1999. Translated from the 1992 German original and with a note by Norbert Schappacher, With a foreword by G. Harder.

[22] O. Timothy O'Meara. Introduction to quadratic forms. Classics in Mathematics. SpringerVerlag, Berlin, 2000. Reprint of the 1973 edition.

[23] M. Peters. Ternäre und quaternäre quadratische Formen und Quaternionenalgebren. Acta Arith., 15:329-365, 1968/1969.

[24] Arnold K. Pizer. Type numbers of Eichler orders. J. Reine Angew. Math., 264:76-102, 1973.

[25] Paul Ponomarev. Arithmetic of quaternary quadratic forms. Acta Arith., 29(1):1-48, 1976.

[26] Paul Ponomarev. Class number formulas for quaternary quadratic forms. Acta Arith., 39(1):95-104, 1981.

[27] I. Reiner. Maximal orders, volume 28 of London Mathematical Society Monographs. New Series. The Clarendon Press Oxford University Press, Oxford, 2003. Corrected reprint of the 1975 original, With a foreword by M. J. Taylor.

[28] Joseph J. Rotman. Advanced modern algebra, volume 114 of Graduate Studies in Mathematics. American Mathematical Society, Providence, RI, 2010. Second edition [of MR2043445].

[29] Peter Stevenhagen. On a problem of Eisenstein. Acta Arith., 74(3):259-268, 1996.

[30] Hiroshi Suzuki. On the capitulation problem. In Class field theory-its centenary and prospect (Tokyo, 1998), volume 30 of Adv. Stud. Pure Math., pages 483-507. Math. Soc. Japan, Tokyo, 2001.

[31] John Tate. Endomorphisms of abelian varieties over finite fields. Invent. Math., 2:134-144, 1966.

[32] Marie-France Vignéras. Nombre de classes d'un ordre d'Eichler et valeur au point -1 de la fonction zêta d'un corps quadratique réel. Enseignement Math. (2), 21(1):69-105, 1975.

[33] Marie-France Vignéras. Invariants numériques des groupes de Hilbert. Math. Ann., 224(3):189-215, 1976.

[34] Marie-France Vignéras. Arithmétique des algèbres de quaternions, volume 800 of Lecture Notes in Mathematics. Springer, Berlin, 1980.

[35] Lawrence C. Washington. Introduction to cyclotomic fields, volume 83 of Graduate Texts in Mathematics. Springer-Verlag, New York, second edition, 1997.

[36] Fu-Tsun Wei and Chia-Fu Yu. Class numbers of central simple algebras over global function fields. Int. Math. Res. Not. IMRN, (11):3525-3575, 2015.

[37] Jiangwei Xue, Tse-Chung Yang, and Chia-Fu Yu. Supersingular abelian surfaces and Eichler's class number formula. ArXiv e-prints, April 2014, arXiv:1404.2978 to appear in The Asian Journal of Mathematics. 
[38] Jiangwei Xue, Tse-Chung Yang, and Chia-Fu Yu. Numerical invariants of totally imaginary quadratic $\mathbb{Z}[\sqrt{p}]$-orders. Taiwanese J. Math., 20(4):723-741, 2016.

[39] Jiangwei Xue, Tse-Chung Yang, and Chia-Fu Yu. On superspecial abelian surfaces over finite fields. Doc. Math., 21:1607-1643, 2016.

[40] Don Zagier. On the values at negative integers of the zeta-function of a real quadratic field. Enseignement Math. (2), 22(1-2):55-95, 1976.

(Xue) Collaborative Innovation Centre of Mathematics, School of Mathematics and Statistics, Wuhan University, Luojiashan, Wuhan, Hubei, 430072, P.R. China.

E-mail address: xue_j@whu.edu.cn

(Yu) Institute of Mathematics, Academia Sinica and NCTS, Astronomy-Mathematics Building, No. 1, Sec. 4, Roosevelt Road, Taipei 10617, TAiWAN.

E-mail address: chiafu@math.sinica.edu.tw 